\title{
STABILITY, WELL-POSEDNESS AND REGULARITY OF THE HOMOGENEOUS LANDAU EQUATION FOR HARD POTENTIALS
}

\author{
NICOLAS FOURNIER AND DANIEL HEYDECKER
}

\begin{abstract}
We establish the well-posedness and some quantitative stability of the spatially homogeneous Landau equation for hard potentials, using some specific Monge-Kantorovich cost, assuming only that the initial condition is a probability measure with a finite moment of order $p$ for some $p>2$. As a consequence, we extend previous regularity results and show that all non-degenerate measure-valued solutions to the Landau equation, with a finite initial energy, immediately admit analytic densities with finite entropy. Along the way, we prove that the Landau equation instantaneously creates Gaussian moments. We also show existence of weak solutions under the only assumption of finite initial energy.
\end{abstract}

\section{INTRODUCTION AND MAIN RESULTS}

1.1. The Landau equation. We study the spatially homogeneous (Fokker-Planck-)Landau equation, which governs the time-evolution of the distribution $f_{t}, t \geq 0$ of velocities in a plasma:

$$
\partial_{t} f_{t}(v)=\frac{1}{2} \operatorname{div}_{v}\left(\int_{\mathbb{R}^{3}} a\left(v-v_{*}\right)\left[f_{t}\left(v_{*}\right) \nabla f_{t}(v)-f_{t}(v) \nabla f_{t}\left(v_{*}\right)\right] \mathrm{d} v_{*}\right)
$$

where $a$ is the nonnegative, symmetric matrix

$$
a(x)=|x|^{2+\gamma} \Pi_{x^{\perp}} ; \quad \Pi_{x^{\perp}}=\mathbf{I}_{3}-\frac{x x^{*}}{|x|^{2}}
$$

and $\gamma \in[-3,1]$ parametrises a range of models, depending on the interactions between particles. While the most physically relevant case is $\gamma=-3$, which models Coulomb interaction, we will study the cases $\gamma \in(0,1]$ of hard potentials, where the Landau equation (11) may be understood as a limit of the Boltzmann equation in the asymptotic of grazing collisions, see Desvillettes [10 and Villani [40, 41].

This equation was studied in detail by Desvillettes and Villani [11, 12, who give results on existence, uniqueness, regularising effects and large-time behavior. Regarding stability, we refer to [15, on which the present work builds. Let us also mention the work of Carrapatoso [5] on exponential convergence to equilibrium, some recent works of $\mathrm{Chen}, \mathrm{Li}$ and $\mathrm{Wu}[7,8$ ] and Morimoto, Pravda-Starov and $\mathrm{Xu}$ 34 extending the regularity results, as well as the recent gradient flow approach by Carrillo, Delgadino, Desvillettes and Wu [6].

1.2. Notation. We denote by $\mathcal{P}\left(\mathbb{R}^{3}\right)$ the set of probability measures on $\mathbb{R}^{3}$, and for $p>0$, we set $\mathcal{P}_{p}$ to be those probability measures with a finite $p^{\text {th }}$ moment: $\mathcal{P}_{p}\left(\mathbb{R}^{3}\right)=\left\{f \in \mathcal{P}\left(\mathbb{R}^{3}\right): m_{p}(f)<\infty\right\}$, where $m_{p}(f)=\int_{\mathbb{R}^{3}}|v|^{p} f(\mathrm{~d} v)<\infty$.

2010 Mathematics Subject Classification. 82C40,60K35.

Key words and phrases. Fokker-Planck-Landau equation, existence, uniqueness, stability, regularity, MongeKantorovitch distance, Wasserstein distance, coupling, stochastic differential equations. 
We will use the following family of transportation costs to measure the distance between two solutions. For $p>0$ and $f, \tilde{f} \in \mathcal{P}_{p}\left(\mathbb{R}^{3}\right)$, we write $\mathcal{H}(f, \tilde{f})$ for the set of all couplings

$$
\mathcal{H}(f, \tilde{f})=\left\{R \in \mathcal{P}\left(\mathbb{R}^{3} \times \mathbb{R}^{3}\right): R \text { has marginals } f \text { and } \tilde{f}\right\} .
$$

With this notation, we define the optimal transportation cost

$$
\mathcal{T}_{p}(f, \tilde{f})=\inf \left\{\int_{\mathbb{R}^{3} \times \mathbb{R}^{3}}\left(1+|v|^{p}+|\tilde{v}|^{p}\right) \frac{|v-\tilde{v}|^{2}}{1+|v-\tilde{v}|^{2}} R(\mathrm{~d} v, \mathrm{~d} \tilde{v}): R \in \mathcal{H}(f, \tilde{f})\right\} .
$$

The form of this optimal transportation cost is key to our stability and uniqueness arguments; the major improvement in Theorem 8 below relies on a negative term which appears due to a Pozvner effect of the prefactor $\left(1+|v|^{p}+|\tilde{v}|^{p}\right)$. Note that for each $p \geq 2$, there is a constant $C>0$ such that $|v-\tilde{v}|^{p} \leq C\left(1+|v|^{p}+|\tilde{v}|^{p}\right) \frac{|v-\tilde{v}|^{2}}{1+|v-\tilde{v}|^{2}}$, so that for $\mathcal{W}_{p}$ the usual Wasserstein distance of order $p$, we have $\mathcal{W}_{p}^{p} \leq C \mathcal{T}_{p}$. It can also be checked that convergence in $\mathcal{W}_{p}$ implies convergence in $\mathcal{T}_{p}$. It follows that both $\mathcal{W}_{p}$ and $\mathcal{T}_{p}$ generate the same topology, equivalent to weak convergence plus convergence of the $p^{\text {th }}$ moments.

We will also consider regularity of solutions. For $k, s \geq 0$, we define the weighted Sobolev norm

$$
\|u\|_{H_{s}^{k}\left(\mathbb{R}^{3}\right)}^{2}=\sum_{|\alpha| \leq k} \int_{\mathbb{R}^{3}}\left|\partial_{\alpha} u(v)\right|^{2}\left(1+|v|^{2}\right)^{s / 2} \mathrm{~d} v
$$

and the weighted Sobolev space $H_{s}^{k}\left(\mathbb{R}^{3}\right)$ for those $u$ where this is finite. By an abuse of notation, we say that $f \in \mathcal{P}\left(\mathbb{R}^{3}\right)$ belongs to $H_{s}^{k}\left(\mathbb{R}^{3}\right)$ if $f$ admits a density $u$ with respect to the Lebesgue measure with $u \in H_{s}^{k}\left(\mathbb{R}^{3}\right)$, and in this case we write $\|f\|_{H_{s}^{k}\left(\mathbb{R}^{3}\right)}=\|u\|_{H_{s}^{k}\left(\mathbb{R}^{3}\right)}$. Similarly, we say that $f \in \mathcal{P}\left(\mathbb{R}^{3}\right)$ is analytic if $f$ admits an analytic density.

We finally define the entropy $H(f)$ of a probability measure $f \in \mathcal{P}\left(\mathbb{R}^{3}\right)$ by

$$
H(f)= \begin{cases}\int_{\mathbb{R}^{3}} u(v) \log u(v) \mathrm{d} v & \text { if } f \text { has a density } u \\ \infty & \text { otherwise. }\end{cases}
$$

1.3. Weak solutions. We define, for $x \in \mathbb{R}^{3}$,

$$
b(x)=\operatorname{div} a(x)=-2|x|^{\gamma} x .
$$

For $\left(f_{t}\right)_{t \geq 0}$ a family of probability measures on $\mathbb{R}^{3}$ and for $p, q>0$, we say that $\left(f_{t}\right)_{t \geq 0}$ belongs to $L_{l o c}^{\infty}\left([0, \infty), \mathcal{P}_{p}\left(\mathbb{R}^{3}\right)\right) \cap L_{l o c}^{1}\left([0, \infty), \mathcal{P}_{q}\left(\mathbb{R}^{3}\right)\right)$ if

$$
\sup _{t \in[0, T]} m_{p}\left(f_{t}\right)+\int_{0}^{T} m_{q}\left(f_{t}\right) \mathrm{d} t<\infty \quad \text { for all } T>0 .
$$

We will use the following classical notion of weak solutions, see Villani [40] and Goudon [23].

Definition 1. Let $\gamma \in(0,1]$. We say that $\left(f_{t}\right)_{t \geq 0}$ is a weak solution to (1) if it belongs to $L_{\text {loc }}^{\infty}\left([0, \infty), \mathcal{P}_{2}\left(\mathbb{R}^{3}\right)\right) \cap L_{\text {loc }}^{1}\left([0, \infty), \mathcal{P}_{2+\gamma}\left(\mathbb{R}^{3}\right)\right)$, if $m_{2}\left(f_{t}\right) \leq m_{2}\left(f_{0}\right)$ for all $t \geq 0$ and if for all $\varphi \in C_{b}^{2}\left(\mathbb{R}^{3}\right)$, all $t \geq 0$,

$$
\int_{\mathbb{R}^{3}} \varphi(v) f_{t}(\mathrm{~d} v)=\int_{\mathbb{R}^{3}} \varphi(v) f_{0}(\mathrm{~d} v)+\int_{0}^{t} \int_{\mathbb{R}^{3}} \int_{\mathbb{R}^{3}} \mathcal{L} \varphi\left(v, v_{*}\right) f_{s}\left(\mathrm{~d} v_{*}\right) f_{s}(\mathrm{~d} v) \mathrm{d} s,
$$

where

$$
\mathcal{L} \varphi\left(v, v_{*}\right)=\frac{1}{2} \sum_{k, \ell=1}^{3} a_{k \ell}\left(v-v_{*}\right) \partial_{k \ell}^{2} \varphi(v)+\sum_{k=1}^{3} b_{k}\left(v-v_{*}\right) \partial_{k} \varphi(v)
$$


Since $\left|\mathcal{L} \varphi\left(v, v_{*}\right)\right| \leq C_{\varphi}\left(1+|v|+\left|v_{*}\right|\right)^{2+\gamma}$ for $\varphi \in C_{b}^{2}\left(\mathbb{R}^{3}\right)$, every term makes sense in (3).

1.4. Existence and properties of weak solutions. First we summarise some results of Desvillettes and Villani.

Theorem 2 (Desvillettes \& Villani, Theorems 3 and 6 in [1]). Fix $\gamma \in(0,1], p \geq 2$ and $f_{0} \in$ $\mathcal{P}_{p}\left(\mathbb{R}^{3}\right)$.

(a) If $\left(f_{t}\right)_{t \geq 0}$ is any weak solution to (11) starting at $f_{0}$, then we have conservation of the kinetic energy, i.e. $m_{2}\left(f_{t}\right)=m_{2}\left(f_{0}\right)$ for all $t \geq 0$, and the estimates $\sup _{s \in[0, \infty)} m_{p}\left(f_{s}\right)<\infty$ and $\int_{0}^{t} m_{p+\gamma}\left(f_{s}\right) \mathrm{d} s<\infty$ for all $t \geq 0$. Further, for all $q>0$ and $t_{0}>0, \sup _{t \geq t_{0}} m_{q}\left(f_{t}\right)<\infty$.

(b) If $p>2$, then a weak solution starting at $f_{0}$ exists.

(c) If $p>2$ and if $f_{0}$ is not concentrated on a line, then there exists a weak solution $\left(f_{t}\right)_{t \geq 0}$ starting at $f_{0}$ such that for all $t>0, f_{t}$ has finite entropy $H\left(f_{t}\right)<\infty$ and

$$
\text { for all } k, s \geq 0 \text { and all } t_{0}>0, \quad \sup _{t \geq t_{0}}\left\|f_{t}\right\|_{H_{s}^{k}\left(\mathbb{R}^{3}\right)}<\infty .
$$

Let us remark that the cited theorem makes the additional assumption in (a) that $f_{t}$ has a density for all $t \geq 0$, but this is not used in the proof. Regarding (b), the cited theorem assumes that $f_{0}$ has a density, but this is only required to show that the weak solution they build has a density, for all times. Concerning (c), Desvillettes and Villani also assume that $f_{0}$ has a density, but only use that $f_{0}$ is not concentrated on a line, see the remark under Lemma 9 of the cited work. To be more explicit, $f_{0}$ not concentrated on a line means that for any $x_{0}, u_{0} \in \mathbb{R}^{3}$, setting $L=\left\{x_{0}+\lambda u_{0}: \lambda \in \mathbb{R}\right\}$, there holds $f_{0}\left(\mathbb{R}^{3} \backslash L\right)>0$.

Regarding existence, we are able to prove the following extension to (b) above, removing the condition that $f_{0} \in \mathcal{P}_{p}\left(\mathbb{R}^{3}\right)$ for some $p>2$ and requiring only $f_{0} \in \mathcal{P}_{2}\left(\mathbb{R}^{3}\right)$.

Theorem 3. Let $\gamma \in(0,1]$ and $f_{0} \in \mathcal{P}_{2}\left(\mathbb{R}^{3}\right)$. There exists a weak solution to (1) starting at $f_{0}$.

Let us now state the following strengthening of (c), due to Chen, $\mathrm{Li}$ and $\mathrm{Xu}$.

Theorem 4 (Chen, Li \& Xu, Theorem 1.1 in [8]). Fix $\gamma \in(0,1]$. Let $\left(f_{t}\right)_{t \geq 0}$ be a weak solution to (11) such that the estimate (5) holds. Then $f_{t}$ is analytic for all $t>0$.

Our main result on regularity is as follows, and shows that the conclusions above apply to all weak solutions to (1), aside from the degenerate case of point masses.

Theorem 5. Fix $\gamma \in(0,1]$. Let $f_{0} \in \mathcal{P}_{2}\left(\mathbb{R}^{3}\right)$ be a probability measure which is not a Dirac mass, and let $\left(f_{t}\right)_{t \geq 0}$ be any weak solution to (10) starting at $f_{0}$. Then the estimate (5) holds and for all $t>0, f_{t}$ is analytic and has a finite entropy.

We emphasise that Theorem 5 applies to any weak solution, while Theorems 2 -(c) and 4 only show that there exists such a regular solution (see the remarks after Theorem 6 in [11). This follows from Theorem 8 below, although we are not able to prove uniqueness under the sole assumption that $f_{0} \in \mathcal{P}_{2}\left(\mathbb{R}^{3}\right)$. Let us also remark that, in the excluded case where $f_{0}=\delta_{v_{0}}$ is a point mass, then the unique solution is $f_{t}=\delta_{v_{0}}$ for all $t \geq 0$ by conservation of energy and momentum, and so there is no hope of regularity.

As a step towards our main stability result below, we will prove the following proposition, which improves on the appearance of moments in item (a) above and may be of independent interest. 
Proposition 6. Fix $\gamma \in(0,1]$ and consider a weak solution $\left(f_{t}\right)_{t \geq 0}$ to (11). There are some constants $a>0$ and $C>0$, both depending only on $\gamma$ and $m_{2}\left(f_{0}\right)$, such that

$$
\int_{\mathbb{R}^{3}} e^{a|v|^{2}} f_{t}(\mathrm{~d} v) \leq C \exp \left[C t^{-2 / \gamma}\right] \text { for all } t>0 .
$$

Since the preliminary version of this work, the first author has studied the Boltzmann equation with hard potentials and without cutoff, which produces some exponential moments of the form $\int_{\mathbb{R}^{3}} e^{a|v|^{\rho}} f_{t}(d v)$, with $\rho \in(\gamma, 2]$ depending on the singularity of the angular collision kernel. In the case with cutoff, only exponential moments of the form $\int_{\mathbb{R}^{3}} e^{a|v|^{\gamma}} f_{t}(d v)$ become finite for $t>0$, see Alonso-Gamba-Taskovic [2].

1.5. Uniqueness and stability. Let us mention the following result, due to the first author and Guillin, which can be compared to our result and on which we build.

Theorem 7 (Fournier \& Guillin, Theorem 2 in [15). Fix $\gamma \in(0,1]$ and let $f_{0} \in \mathcal{P}\left(\mathbb{R}^{3}\right)$ be such that

$$
\mathcal{E}_{\alpha}\left(f_{0}\right)=\int_{\mathbb{R}^{3}} e^{|v|^{\alpha}} f_{0}(\mathrm{~d} v)<\infty \text { for some } \alpha>\gamma .
$$

Then there exists a unique weak solution $\left(f_{t}\right)_{t \geq 0}$ to (11) starting at $f_{0}$. Moreover, if $\eta \in(0,1)$, $\lambda \in(0, \infty)$ and $T>0$, then there exists a constant $C=C\left(T, \eta, \mathcal{E}_{\alpha}\left(f_{0}\right), \lambda\right)$ such that, if $\left(\tilde{f}_{t}\right)_{t \geq 0}$ is another solution satisfying $\sup _{t \in[0, T]} m_{2+\gamma}\left(\tilde{f}_{t}\right) \leq \lambda$, then

$$
\sup _{t \in[0, T]} \mathcal{W}_{2}\left(f_{t}, \tilde{f}_{t}\right) \leq C\left[\mathcal{W}_{2}\left(f_{0}, \tilde{f}_{0}\right)\right]^{1-\eta}
$$

where $\mathcal{W}_{2}$ is the usual Wasserstein distance with quadratic cost.

The main result of this paper is the following, which consists in relaxing the condition (6) and in replacing, via another transportation cost, the Hölder dependance in the initial condition by some Lipschitz dependance.

Theorem 8. Fix $\gamma \in(0,1]$ and $p>2$ and two weak solutions $\left(f_{t}\right)_{t \geq 0}$ and $\left(\tilde{f}_{t}\right)_{t \geq 0}$ to (11) starting from $f_{0}$ and $\tilde{f}_{0}$, both belonging to $\mathcal{P}_{p}\left(\mathbb{R}^{3}\right)$. There is a constant $C$, depending only on $p$ and $\gamma$, such that for all $t \geq 0$,

$$
\mathcal{T}_{p}\left(f_{t}, \tilde{f}_{t}\right) \leq \mathcal{T}_{p}\left(f_{0}, \tilde{f}_{0}\right) \exp \left(C\left[1+\sup _{s \in[0, t]} m_{p}\left(f_{s}+\tilde{f}_{s}\right)\right]\left[1+\int_{0}^{t}\left(1+m_{p+\gamma}\left(f_{s}+\tilde{f}_{s}\right)\right) \mathrm{d} s\right]\right) .
$$

Together with Theorem 2, this shows that when $f_{0} \in \mathcal{P}_{p}\left(\mathbb{R}^{3}\right)$ for some $p>2$, (1) has a unique weak solution and this provides a quantitative stability estimate.

1.6. Discussion. This current paper is primarily concerned with stability, continuing the previous analyses of the Cauchy problem for the Landau equation with hard potentials by Arsen'ev-Buryak [3], Desvillettes-Villani [11, see also [15. In addition to the mathematical interest of uniqueness and stability, these are physically relevant criteria: if the equation is not well-posed, then it cannot be a complete description of the system and additional information is needed. Let us also note that stability estimates play a key role in the functional framework of Mischler-Mouhot [31] and Mischler-Mouhot-Wennberg 32 for proving propagation of chaos for interacting particle systems, and these have been applied to Kac's process [30]. See also the work of Norris [36] and [28. In this context, it is particularly advantageous that our result requires neither regularity nor exponential moments, as these are not readily applicable to the empirical measures of the particle system. It 
is also satisfying to get a Lipschitz dependance in the initial condition, so that error terms will not increase too much as time evolves.

The study of stability via coupling, on which this work builds, goes back to Tanaka 38 for the Boltzmann equation in the case of Maxwell molecules; let us mention the later works [18, 17, 29] which apply the same principle in the context of hard potentials. The same idea was applied to the Landau equation by Funaki 21] and has previously been applied by the first author [15] in the context of stability and propagation of chaos. See [19] for a review of coupling methods for PDEs.

Compared to the previous literature regarding uniqueness and stability for the Landau equation with hard potentials, our main result is substantially stronger and more general. The uniqueness result of Desvillettes and Villani [11] requires that the initial data $f_{0}$ has a density $u_{0}$ satisfying

$$
\int_{\mathbb{R}^{3}}\left(1+|v|^{2}\right)^{p / 2} u_{0}^{2}(v) \mathrm{d} v<\infty \quad \text { for some } p>15+5 \gamma,
$$

while the result of the $[15$ recalled in Theorem 7 above allows measure solutions, but requires a finite exponential moment. Our result therefore allows much less localisation than either of the results above, while also not requiring any regularity on the initial data $f_{0}, \tilde{f}_{0}$.

The case $\gamma=0$ of Maxwell molecules is particularly simple, and results of Villani [39] show existence and uniqueness only assuming finite energy, i.e. that $f_{0} \in \mathcal{P}_{2}\left(\mathbb{R}^{3}\right)$. Note that the Boltzmann equation for hard potentials with cutoff has also been shown to be well-posed by Mischler-Wennberg [33] as soon as $f_{0} \in \mathcal{P}_{2}\left(\mathbb{R}^{3}\right)$, by a completely different method breaking down in the case without cutoff. Hence our condition on $f_{0}$ for Theorem 8 , namely the finiteness of a moment of order $p>2$, seems almost optimal. While this is feasable for existence, we did not manage to prove uniqueness assuming only a finite initial energy.

To summarize, our uniqueness and stability statement Theorem 8 is much stronger than the previous results and almost optimal, since we assume that $f_{0} \in \mathcal{P}_{2+}\left(\mathbb{R}^{3}\right)$ instead of (8) as in [1] or (6) as in [15]; we slightly improve in Theorem 3 the existence result of [11, assuming that $f_{0} \in \mathcal{P}_{2}\left(\mathbb{R}^{3}\right)$ instead of $f_{0} \in \mathcal{P}_{2+}\left(\mathbb{R}^{3}\right)$; we are able to prove in Theorem 5 the smoothness of any weak solution with $f_{0} \in \mathcal{P}_{2}\left(\mathbb{R}^{3}\right)$, instead of showing the existence of one smooth solution when $f_{0} \in \mathcal{P}_{2+}\left(\mathbb{R}^{3}\right)$ as in $[11$ and $[\underline{8}$; and we prove the appearance of some Gaussian moments for any weak solution with $f_{0} \in \mathcal{P}_{2}\left(\mathbb{R}^{3}\right)$.

Let us finally mention that in the case $\gamma=0$, a stronger 'ulta-analytic' regularity is known, see Morimoto, Pravda-Starov and Xu [34]; in this case, one has the advantage that the coefficients of (11) are already analytic (polynomial) functions. Another approach to regularity results similar is the use of Malliavin calculus, see Guérin [24.

Finally, let us mention the current theory for other Landau equations. In the case of soft potentials $\gamma \in(-3,0)$, we refer to [14, 16]. The Coulomb case $\gamma=-3$, which is most directly physically relevant and significantly more difficult, has also received significant attention, including by Villani 40, Desvillettes 9 and the first author [13. Let us mention a number of works by Guo [26], He-Yang [27, Golse-Imbert-Mouhot-Vasseur [22] and Mouhot [35] on the Cauchy problem for the full, spatially inhomogeneous, Landau equation. Finally, the Landau-Fermi-Dirac equation has been recently studied by Alonso, Bagland and Lods [1].

1.7. Strategy. We emphasise that the main result is the stability and uniqueness result Theorem 8 Theorem [5 about regularity will then follow from previous works. For Theorem 8 , our strategy is to build on the techniques of [15], [36] and [29]. The key new idea is a Povzner-type inequality [37. Considering a weighted cost of the form $\left(1+|v|^{p}+|\tilde{v}|^{p}\right)|v-\tilde{v}|^{2}$ instead of $|v-\tilde{v}|^{2}$, an 
additional, negative 'Povzner term' arises which produces an advantageous cancelation and allows us to use a Grönwall inequality. We rather study (at the price of technical difficulties) the cost $\left(1+|v|^{p}+|\tilde{v}|^{p}\right)|v-\tilde{v}|^{2} /\left(1+|v-\tilde{v}|^{2}\right)$, because it requires less moments to be well-defined.

In the case of the Boltzmann equation for hard potentials without cutoff [29], this technique leads to stability under the assumption only of some $p^{\text {th }}$ moment, for some computable, but potentially large, $p$, improving on previous results which required exponential moments [18. In the case of the Landau equation, the calculations become more tractable; we find explicit, rather than explicitable constants, and are able to use tricks of [15] and [16. In this context, we seek to minimise the number $p$ of moments required, and very delicate calculations are needed, see Lemma 13 and its proof, to allow for any $p>2$.

1.8. Plan of the Paper. The paper is structured as follows. In Section 2, we will present some preliminary calculations which are used throughout the paper. In Section 3 we will prove some useful moment properties, including Proposition 6.

Section 4- 5 are devoted to the proof of our stability result Theorem 8 Section 4 introduces the Tanaka-style coupling and presents the key estimate without proof. This allows us to prove Theorem 8 in Section 5 , and we finally return to prove the central estimate in Section 8 , Informally, the main important points of the proof are Proposition 10, where we introduce the coupling between two given weak solutions, Lemmas 12 and 13, containing the central computation, and Lemma 14 where we establish the stability estimate (7) under some additional conditions. Since the proof of the central computation Lemma 13 is rather technical, it is deferred until Section 8 .

Section [6] consists of a self-contained proof of our existence result Theorem 3, building only on Theorem 2 and using the de La Vallée Poussin theorem and a compactness argument.

In Section 7 we prove Theorem $[5$ about smoothness. We show a very mild regularity result (Lemma 16): solutions do not remain concentrated on lines. This allows us to apply Theorems 2-(c) and 4, exploiting the uniqueness from Theorem 8 .

Finally, Section 8 contains the proof of the estimate Lemma 13 .

Acknowledgements. The second author is supported by the UK Engineering and Physical Sciences Research Council (EPSRC) grant EP/L016516/1 for the University of Cambridge Centre for Doctoral Training, the Cambridge Centre for Analysis.

\section{Preliminaries}

We introduce a few notation and handle some computations of constant use. We denote by $|\cdot|$ the Euclidean norm on $\mathbb{R}^{3}$ and for $A$ and $B$ two $3 \times 3$ matrices, we put $\|A\|^{2}=\operatorname{Tr}\left(A A^{*}\right)$ and $\left\langle\langle A, B\rangle=\operatorname{Tr}\left(A B^{*}\right)\right.$.

2.1. A few estimates of the parameters of the Landau equation. For $x \in \mathbb{R}^{3}$, we introduce

$$
\sigma(x)=[a(x)]^{1 / 2}=|x|^{1+\gamma / 2} \Pi_{x^{\perp}} .
$$

For $x, \tilde{x} \in \mathbb{R}^{3}$, it holds that

(9) $\|\sigma(x)\|^{2}=2|x|^{\gamma+2}$ and $\left\langle\langle\sigma(x), \sigma(\tilde{x})\rangle=|x|^{1+\gamma / 2}|\tilde{x}|^{1+\gamma / 2}\left(1+\frac{(x \cdot \tilde{x})^{2}}{|x|^{2}|\tilde{x}|^{2}}\right) \geq 2|x|^{\gamma / 2}|\tilde{x}|^{\gamma / 2}(x \cdot \tilde{x})\right.$.

Indeed, it suffices to justify the second assertion, and a simple computation shows that $\Pi_{x^{\perp}} \Pi_{\tilde{x}^{\perp}}=$ $\mathbf{I}_{3}-|x|^{-2} x x^{*}-|\tilde{x}|^{-2} \tilde{x} \tilde{x}^{*}+|x|^{-2}|\tilde{x}|^{-2}(x \cdot \tilde{x}) x \tilde{x}^{*}$, from which we conclude that

$$
\left\langle\langle\sigma(x), \sigma(\tilde{x})\rangle=|x|^{1+\gamma / 2}|\tilde{x}|^{1+\gamma / 2} \operatorname{Tr}\left(\Pi_{x^{\perp}} \Pi_{\tilde{x}^{\perp}}\right)=|x|^{1+\gamma / 2}|\tilde{x}|^{1+\gamma / 2}\left[1+|x|^{-2}|\tilde{x}|^{-2}(x \cdot \tilde{x})^{2}\right],\right.
$$


which is greater than $2|x|^{\gamma / 2}|\tilde{x}|^{\gamma / 2}(x \cdot \tilde{x})$ because $1+a^{2} \geq 2 a$.

For $a, b \geq 0$ and $\alpha \in(0,1)$, there holds

$$
\left|a^{\alpha}-b^{\alpha}\right| \leq(a \vee b)^{\alpha-1}|a-b| .
$$

Indeed, if e.g. $a \geq b$, then $a^{\alpha}-b^{\alpha}=a^{\alpha}\left[1-(b / a)^{\alpha}\right] \leq a^{\alpha}(1-b / a)=a^{\alpha-1}(a-b)$.

For $x, \tilde{x} \in \mathbb{R}^{3}$, recalling that $b(x)=-2|x|^{\gamma} x$, we have

$$
|b(x)-b(\tilde{x})| \leq 2|x|^{\gamma}|x-\tilde{x}|+\left.2|\tilde{x}||| x\right|^{\gamma}-|\tilde{x}|^{\gamma}\left|\leq 2\left(|x|^{\gamma}+|\tilde{x}|^{\gamma}\right)\right| x-\tilde{x} \mid,
$$

because $|\tilde{x}||x|^{\gamma}-|\tilde{x}|^{\gamma}|\leq| \tilde{x}\left|(|x| \vee|\tilde{x}|)^{\gamma-1}\right| x-\left.\tilde{x}|\leq| \tilde{x}\right|^{\gamma}|x-\tilde{x}|$ by (10). We also have, thanks to (91),

$$
\| \sigma(x)-\left.\sigma(\tilde{x})\right|^{2} \leq 2|x|^{\gamma+2}+2|\tilde{x}|^{\gamma+2}-4|x|^{\gamma / 2}|\tilde{x}|^{\gamma / 2}(x \cdot \tilde{x})=\left.2|| x\right|^{\gamma / 2} x-\left.|\tilde{x}|^{\gamma / 2} \tilde{x}\right|^{2} .
$$

Proceeding as for (11), we deduce that

$$
\| \sigma(x)-\left.\sigma(\tilde{x})\right|^{2} \leq 2\left(|x|^{\gamma / 2}|x-\tilde{x}|+\left.|\tilde{x}||| x\right|^{\gamma / 2}-|\tilde{x}|^{\gamma / 2} \mid\right)^{2} \leq 2\left(|x|^{\gamma / 2}+|\tilde{x}|^{\gamma / 2} \mid\right)^{2}|x-\tilde{x}|^{2} .
$$

Finally, for $v, v_{*} \in \mathbb{R}^{3}, \sigma\left(v-v_{*}\right) v=\sigma\left(v-v_{*}\right) v_{*}$, because $\Pi_{\left(v-v_{*}\right)}\left(v-v_{*}\right)=0$, and so

$$
\left|\sigma\left(v-v_{*}\right) v\right| \leq C|| \sigma\left(v-v_{*}\right)||\left(|v| \wedge\left|v_{*}\right|\right) \leq C\left|v-v_{*}\right|^{1+\gamma / 2}\left(|v| \wedge\left|v_{*}\right|\right) \leq C\left|v-v_{*}\right|^{\gamma / 2}|v|\left|v_{*}\right|,
$$

because $\left|v-v_{*}\right|\left(|v| \wedge\left|v_{*}\right|\right) \leq\left(|v|+\left|v_{*}\right|\right)\left(|v| \wedge\left|v_{*}\right|\right) \leq 2|v|\left|v_{*}\right|$.

2.2. Transport costs. For technical reasons, we will have to play with a larger family of transport costs. For $p>0$ and $\varepsilon>0$, for $f, \tilde{f} \in \mathcal{P}_{p}\left(\mathbb{R}^{3}\right)$, we define

$$
\mathcal{T}_{p, \varepsilon}(f, \tilde{f})=\inf \left\{\int_{\mathbb{R}^{3} \times \mathbb{R}^{3}} c_{p, \varepsilon}(v, \tilde{v}) R(\mathrm{~d} v, \mathrm{~d} \tilde{v}): R \in \mathcal{H}(f, \tilde{f})\right\},
$$

where

$$
c_{p, \varepsilon}(v, \tilde{v})=\left(1+|v|^{p}+|\tilde{v}|^{p}\right) \varphi_{\varepsilon}\left(|v-\tilde{v}|^{2}\right) \quad \text { and } \quad \varphi_{\varepsilon}(r)=\frac{r}{1+\varepsilon r} .
$$

We have $\mathcal{T}_{p}=\mathcal{T}_{p, 1}$. This definition also makes sense in the case $\varepsilon=0, \phi_{0}(r)=r$, in which case we require $f, \tilde{f} \in \mathcal{P}_{p+2}\left(\mathbb{R}^{3}\right)$ for the integral to be well-defined. In either case, it is straightforward to see that there exists a coupling attaining the infimum; we refer to Villani [42] for many details of such costs. Since $\varphi_{\varepsilon}^{\prime}(r)=(1+\varepsilon r)^{-2}$ and $\varphi_{\varepsilon}^{\prime \prime}(r)=-2 \varepsilon(1+\varepsilon r)^{-3}$,

$$
r \varphi_{\varepsilon}^{\prime}(r) \leq \varphi_{\varepsilon}(r), \quad 0 \leq \varphi_{\varepsilon}^{\prime}(r) \leq 1 \quad \text { and } \quad \varphi_{\varepsilon}^{\prime \prime}(r) \leq 0 .
$$

Let us remark that the cost $c_{p, \varepsilon}$ satisfies a relaxed triangle inequality: for some $C>0$ depending only on $p>0$ and $\varepsilon \geq 0$, for all $v, w, y \in \mathbb{R}^{3}$,

$$
c_{p, \varepsilon}(v, y) \leq C\left[c_{p, \varepsilon}(v, w)+c_{p, \varepsilon}(w, y)\right] .
$$

The case where $\varepsilon=0$ was treated in [29, Section 2]. If now $\varepsilon>0, \frac{1}{2}\left(\varepsilon^{-1} \wedge r\right) \leq \varphi_{\varepsilon}(r) \leq\left(\varepsilon^{-1} \wedge r\right)$, so that it suffices to prove (17) with the cost $c_{p, \varepsilon}(v, \tilde{v})$ replaced by $\left(1+|v|^{p}+|\tilde{v}|^{p}\right)\left(|v-\tilde{v}|^{2} \wedge \varepsilon^{-1}\right)$. This can be deduced from the case where $\varepsilon=0$, case-by-case, depending on which of $|v-w|^{2}$, $|w-y|^{2},|v-y|^{2}$ are less than $\varepsilon^{-1}$.

It follows that the optimal transportation costs $\mathcal{T}_{p, \varepsilon}$ are semimetrics in that one replaces the usual triangle inequality with the bound, for all $f, g, h \in \mathcal{P}_{p}$,

$$
\mathcal{T}_{p, \varepsilon}(f, h) \leq C\left[\mathcal{T}_{p, \varepsilon}(f, g)+\mathcal{T}_{p, \varepsilon}(g, h)\right] .
$$




\section{Moment Properties of the Landau Equation}

This section is devoted to some moment estimates. We start with the appearance of Gaussian moments, following the strategy introduced by Bobylev [4 for the Boltzmann equation.

Proof of Proposition [6. We consider any weak solution $\left(f_{t}\right)_{t \geq 0}$ to (11). By Theorem 2, we know that $m_{2}\left(f_{t}\right)=m_{2}\left(f_{0}\right)$ for all $t \geq 0$. If $m_{2}\left(f_{0}\right)=0$, we deduce that $f_{t}=\delta_{0}$ for all $t>0$, so the result is obvious. We thus assume that $m_{2}\left(f_{0}\right)>0$ and, by scaling, that $m_{2}\left(f_{0}\right)=1$. During the proof, $C$ will denote a constant which may only depend on $\gamma$, but may vary from line to line.

Step 1. Here we prove that for all $p \geq 2$, all $t>0$,

$$
\frac{d}{d t} m_{p}\left(f_{t}\right) \leq-p m_{p+\gamma}\left(f_{t}\right)+p m_{p}\left(f_{t}\right)+C p^{2}\left[m_{p-2+\gamma}\left(f_{t}\right)+m_{p-2}\left(f_{t}\right) m_{2+\gamma}\left(f_{t}\right)\right] .
$$

By Theorem 2, we know that for all $q>0$, all $t_{0}>0, \sup _{t \geq t_{0}} m_{q}\left(f_{t}\right)<\infty$, so that we can apply (3) with $\varphi(v)=|v|^{p}$ on $\left[t_{0}, \infty\right)$. We deduce that $m_{p}\left(f_{t}\right)$ is of class $C^{1}$ on $(0, \infty)$ and get

$$
\frac{d}{d t} m_{p}\left(f_{t}\right)=\int_{\mathbb{R}^{3}} \int_{\mathbb{R}^{3}} \mathcal{L} \varphi\left(v, v_{*}\right) f_{t}\left(\mathrm{~d} v_{*}\right) f_{t}(\mathrm{~d} v) \text { for all } t>0 .
$$

Since $\varphi(v)=|v|^{p}=\left(v_{1}^{2}+v_{2}^{2}+v_{3}^{2}\right)^{p / 2}$, we have

$$
\partial_{k} \varphi(v)=p|v|^{p-2} v_{k} \quad \text { and } \quad \partial_{k \ell}^{2} \varphi(v)=p|v|^{p-2} \mathbb{I}_{\{k=\ell\}}+p(p-2)|v|^{p-4} v_{k} v_{\ell}
$$

We set $x=v-v_{*}$ and note that, since $\sigma(x)=[a(x)]^{1 / 2}$ is symmetric, $\sum_{k, \ell=1}^{3} a_{k \ell}(x) \mathbb{I}_{\{k=\ell\}}=$ $\operatorname{Tr} a(x)=\|\sigma(x)\|^{2}$ and $\sum_{k, \ell=1}^{3} a_{k \ell}(x) v_{k} v_{\ell}=\sum_{k, \ell, j=1}^{3} \sigma_{k j}(x) \sigma_{\ell j}(x) v_{k} v_{\ell}=|\sigma(x) v|^{2}$. Thus

$$
\mathcal{L} \varphi\left(v, v_{*}\right)=p|v|^{p-2} v \cdot b(x)+\frac{p}{2}|v|^{p-2}|| \sigma(x)||^{2}+\frac{p(p-2)}{2}|v|^{p-4}|\sigma(x) v|^{2} .
$$

Recalling that $b(x)=-2|x|^{\gamma} x$ and that $\|\sigma(x)\|^{2}=2|x|^{\gamma+2}$ by (9), we have

$$
v \cdot b(x)+\frac{1}{2}\|\sigma(x)\|^{2}=-2|x|^{\gamma}\left(v-v_{*}\right) \cdot v+|x|^{\gamma}\left(|v|^{2}+\left|v_{*}\right|^{2}-2 v \cdot v_{*}\right)=-|x|^{\gamma}|v|^{2}+|x|^{\gamma}\left|v_{*}\right|^{2} .
$$

Since moreover $|\sigma(x) v| \leq C|x|^{\gamma / 2}|v|\left|v_{*}\right|$ by (14), we find that

$$
\mathcal{L} \varphi\left(v, v_{*}\right) \leq-p|x|^{\gamma}|v|^{p}+C p^{2}|x|^{\gamma}|v|^{p-2}\left|v_{*}\right|^{2} .
$$

Using now that $|x|^{\gamma} \geq|v|^{\gamma}-\left|v_{*}\right|^{\gamma}$ and that $|x|^{\gamma} \leq|v|^{\gamma}+\left|v_{*}\right|^{\gamma}$, we conclude that

$$
\mathcal{L} \varphi\left(v, v_{*}\right) \leq-p|v|^{p+\gamma}+p|v|^{p}\left|v_{*}\right|^{\gamma}+C p^{2}\left(|v|^{p-2+\gamma}\left|v_{*}\right|^{2}+|v|^{p-2}\left|v_{*}\right|^{2+\gamma}\right) .
$$

Plugging this into (19), we find that

$$
\frac{d}{d t} m_{p}\left(f_{t}\right) \leq-p m_{p+\gamma}\left(f_{t}\right)+p m_{p}\left(f_{t}\right) m_{\gamma}\left(f_{t}\right)+C p^{2}\left(m_{p-2+\gamma}\left(f_{t}\right) m_{2}\left(f_{t}\right)+m_{p-2}\left(f_{t}\right) m_{2+\gamma}\left(f_{t}\right)\right) .
$$

The conlusion follows, since $m_{\gamma}\left(f_{t}\right) \leq\left[m_{2}\left(f_{t}\right)\right]^{\gamma / 2}=1$.

Step 2. We now deduce that for all $p \geq 4$,

$$
\frac{d}{d t} m_{p}\left(f_{t}\right) \leq-p\left[m_{p}\left(f_{t}\right)\right]^{1+\gamma /(p-2)}+p m_{p}\left(f_{t}\right)+C p^{2}\left[m_{p}\left(f_{t}\right)\right]^{1-(2-\gamma) /(p-2)} .
$$

For any $\beta>\alpha \geq 2$, since $|v|^{2} f_{t}(\mathrm{~d} v)$ is a probability measure,

$$
m_{\alpha}\left(f_{t}\right)=\int_{\mathbb{R}^{3}}|v|^{\alpha-2}|v|^{2} f_{t}(\mathrm{~d} v) \leq\left(\int_{\mathbb{R}^{3}}|v|^{\beta-2}|v|^{2} f_{t}(\mathrm{~d} v)\right)^{(\alpha-2) /(\beta-2)}=\left[m_{\beta}\left(f_{t}\right)\right]^{(\alpha-2) /(\beta-2)} .
$$


We deduce that $m_{p}\left(f_{t}\right) \leq\left[m_{p+\gamma}\left(f_{t}\right)\right]^{(p-2) /(p+\gamma-2)}$, whence

$$
m_{p+\gamma}\left(f_{t}\right) \geq\left[m_{p}\left(f_{t}\right)\right]^{(p+\gamma-2) /(p-2)}=\left[m_{p}\left(f_{t}\right)\right]^{1+\gamma /(p-2)},
$$

that

and that

$$
m_{p-2+\gamma}\left(f_{t}\right) \leq\left[m_{p}\left(f_{t}\right)\right]^{(p-4+\gamma) /(p-2)},
$$

$$
m_{p-2}\left(f_{t}\right) m_{2+\gamma}\left(f_{t}\right) \leq\left[m_{p}\left(f_{t}\right)\right]^{(p-4) /(p-2)+\gamma /(p-2)}=\left[m_{p}\left(f_{t}\right)\right]^{(p-4+\gamma) /(p-2)} .
$$

This completes the step, since $(p-4+\gamma) /(p-2)=1-(2-\gamma) /(p-2)$.

Step 3. For $u:(0, \infty) \rightarrow(0, \infty)$ of class $C^{1}$ satisfying, for some $a, b, c, \alpha, \beta>0$, for all $t>0$,

$$
u^{\prime}(t) \leq-a[u(t)]^{1+\alpha}+b u(t)+c[u(t)]^{1-\beta},
$$

it holds that

$$
\forall t>0, \quad u(t) \leq\left(\frac{2}{a \alpha t}\right)^{1 / \alpha}+\left(\frac{4 b}{a}\right)^{1 / \alpha}+\left(\frac{4 c}{a}\right)^{1 /(\alpha+\beta)} .
$$

Indeed, we set $h(r)=-a r^{1+\alpha}+b r+c r^{1-\beta}$ and we observe that

$$
h(r) \leq-\frac{a}{2} r^{1+\alpha} \quad \text { for all } r \geq u_{*}=\max \left\{(4 b / a)^{1 / \alpha},(4 c / a)^{1 /(\alpha+\beta)}\right\} .
$$

We now fix $t_{0}>0$.

(a) If $u\left(t_{0}\right) \leq u_{*}$, we have $u(t) \leq u_{*}$ for all $t \geq t_{0}$ because $h\left(u_{*}\right) \leq 0$ and $u^{\prime}(t) \leq h(u(t))$.

(b) If now $u\left(t_{0}\right)>u_{*}$, we set $t_{1}=\inf \left\{t>t_{0}: u(t) \leq u_{*}\right\}$ and observe that for $t \in\left[t_{0}, t_{1}\right)$,

$$
u^{\prime}(t) \leq h(u(t)) \leq-\frac{a}{2}[u(t)]^{1+\alpha}
$$

Integrating this inequality, we conclude that, for all $t \in\left[t_{0}, t_{1}\right)$,

$$
u(t) \leq\left[u^{-\alpha}\left(t_{0}\right)+\frac{a \alpha\left(t-t_{0}\right)}{2}\right]^{-1 / \alpha} \leq\left[\frac{2}{a \alpha\left(t-t_{0}\right)}\right]^{1 / \alpha} .
$$

This implies that $t_{1}$ is finite. Since now $u\left(t_{1}\right)=u_{*}$ by definition, we deduce from (a) that $u(t) \leq u_{*}$ for all $t \geq t_{1}$.

Hence in any case, for any $t_{0}>0$, any $t>t_{0}, u(t) \leq \max \left\{u_{*},\left[2 /\left(a \alpha\left(t-t_{0}\right)\right)\right]^{1 / \alpha}\right\}$. Letting $t_{0} \rightarrow 0$, we deduce that $u(t) \leq \max \left\{u_{*},[2 /(a \alpha t)]^{1 / \alpha}\right\}$ for all $t>0$, which completes the step.

Step 4. Using Step 2 and applying Step 3 with $a=p, b=p, c=C p^{2}, \alpha=\gamma /(p-2)$ and $\beta=(2-\gamma) /(p-2)$, we find that for all $p \geq 4$, all $t>0$,

$$
m_{p}\left(f_{t}\right) \leq\left(\frac{2(p-2)}{p \gamma t}\right)^{(p-2) / \gamma}+4^{(p-2) / \gamma}+(4 C p)^{(p-2) / 2} .
$$

Changing again the value of $C$, we conclude that for all $p \geq 4$, all $t>0$,

$$
m_{p}\left(f_{t}\right) \leq\left(1+\frac{2}{\gamma t}\right)^{p / \gamma}+(C p)^{p / 2}
$$

Step 5. For $a>0$ and $t>0$, we write, using that $m_{0}\left(f_{t}\right)=m_{2}\left(f_{t}\right)=1$,

$$
\int_{\mathbb{R}^{3}} e^{a|v|^{2}} f_{t}(\mathrm{~d} v)=\sum_{k \geq 0} \frac{a^{k} m_{2 k}\left(f_{t}\right)}{k !}=1+a+\sum_{k \geq 2} \frac{a^{k} m_{2 k}\left(f_{t}\right)}{k !} .
$$

By Step 4,

$$
\int_{\mathbb{R}^{3}} e^{a|v|^{2}} f_{t}(\mathrm{~d} v) \leq 1+a+\sum_{k \geq 2} \frac{1}{k !}\left[a^{k}\left(1+\frac{2}{\gamma t}\right)^{2 k / \gamma}+a^{k}(2 C k)^{k}\right]
$$


But $\sum_{k \geq 2}(k !)^{-1}(x k)^{k}<\infty$ if $x<1 / e$ by the Stirling formula. Hence if $a<1 /(2 C e)$,

$$
\int_{\mathbb{R}^{3}} e^{a|v|^{2}} f_{t}(\mathrm{~d} v) \leq 1+a+\exp \left[a\left(1+\frac{2}{\gamma t}\right)^{2 / \gamma}\right]+C .
$$

The conclusion follows.

We next prove some technical uniform integrability property.

Lemma 9. Fix $\gamma \in(0,1]$ and $p>2$. Let $\left(f_{t}\right)_{t \geq 0}$ be a weak solution to (1), with initial moment $m_{p}\left(f_{0}\right)<\infty$. Then, for all $\epsilon>0$, there exists $M<\infty$ such that

$$
\limsup _{t \downarrow 0} \int_{\mathbb{R}^{3}}\left(1+|v|^{p}\right) \mathbb{I}_{\{|v|>M\}} f_{t}(\mathrm{~d} v)<\epsilon .
$$

Proof. Let $\psi: \mathbb{R} \rightarrow[0,1]$ be a smooth function such that $\mathbb{1}_{\{r \leq 1\}} \leq \psi(r) \leq \mathbb{I}_{\{r \leq 2\}}$. Now, for $M \geq 1$, define $\chi_{M}: \mathbb{R}^{3} \rightarrow[0,1]$ by $\chi_{M}(v)=\psi(|v| / M)$; these functions are smooth, and satisfy

$$
|v|\left|\nabla \chi_{M}(v)\right| \leq C ; \quad|v|^{2}\left|\nabla^{2} \chi_{M}(v)\right| \leq C
$$

for some constant $C$, independent of $M$. A rough computation using that $|b(x)| \leq C|x|^{1+\gamma}$ and $\|a(x)\| \leq C|x|^{2+\gamma}$ shows that the smooth functions $\varphi_{M}(v)=\left(1+|v|^{p}\right) \chi_{M}(v)$ satisfy

$$
\begin{aligned}
\left|\mathcal{L} \varphi_{M}\left(v, v_{*}\right)\right| & \leq C\left[\left|b\left(v-v_{*}\right)\right|\left|\nabla \varphi_{M}(v)\right|+|| a\left(v-v_{*}\right)||\left|\nabla^{2} \varphi_{M}(v)\right|\right] \\
& \leq C\left[\left|v-v_{*}\right|^{1+\gamma}\left(1+|v|^{p-1}\right)+\left|v-v_{*}\right|^{2+\gamma}\left(1+|v|^{p-2}\right)\right] \\
& \leq C\left(1+|v|^{p+\gamma}+\left|v_{*}\right|^{p+\gamma}\right)
\end{aligned}
$$

for some $C$ which does not depend on $M$. It follows from (3) that, for all $M$,

$$
\left|\int_{\mathbb{R}^{3}} \varphi_{M}(v)\left(f_{t}-f_{0}\right)(\mathrm{d} v)\right| \leq C \int_{0}^{t} m_{p+\gamma}\left(f_{s}\right) \mathrm{d} s .
$$

Now, fix $\epsilon>0$. Since $m_{p+\gamma}\left(f_{s}\right)$ is locally integrable by Theorem 2, there is $t_{0}>0$ such that for all $t \in\left[0, t_{0}\right]$ and all $M \geq 1$,

$$
\left|\int_{\mathbb{R}^{3}} \varphi_{M}(v)\left(f_{t}-f_{0}\right)(\mathrm{d} v)\right| \leq \frac{\varepsilon}{3}
$$

We next fix $M \geq 1$ such that

$$
\int_{\mathbb{R}^{3}}\left(1+|v|^{p}\right) \mathbb{I}_{\{|v| \geq M / 2\}} f_{0}(\mathrm{~d} v)<\frac{\epsilon}{3} .
$$

For any $t \in\left[0, t_{0}\right]$, any $M^{\prime} \geq M$,

$$
\begin{aligned}
& \int_{\mathbb{R}^{3}}\left(1+|v|^{p}\right) \mathbb{I}_{\left\{M<|v| \leq M^{\prime}\right\}} f_{t}(\mathrm{~d} v) \leq \int_{\mathbb{R}^{3}}\left(\varphi_{M^{\prime}}-\varphi_{M / 2}\right)(v) f_{t}(\mathrm{~d} v) \\
& \quad=\int_{\mathbb{R}^{3}} \varphi_{M^{\prime}}(v)\left(f_{t}-f_{0}\right)(\mathrm{d} v)-\int_{\mathbb{R}^{3}} \varphi_{M / 2}(v)\left(f_{t}-f_{0}\right)(\mathrm{d} v)+\int_{\mathbb{R}^{3}}\left(\varphi_{M^{\prime}}-\varphi_{M / 2}\right)(v) f_{0}(\mathrm{~d} v) \leq \varepsilon .
\end{aligned}
$$

For the two first terms, we used (22), while for the last term, we used that $\left(\varphi_{M^{\prime}}-\varphi_{M / 2}\right)(v) \leq$ $\left(1+|v|^{p}\right) \mathbb{1}_{\{|v| \geq M / 2\}}$ and (23). Taking the limit $M^{\prime} \rightarrow \infty$ now gives the result. 


\section{Tanaka-Style Coupling of Landau Processes}

In the spirit of Tanaka [38] for the Boltzmann equation, see Funaki [21] and Guérin [25] for the Landau equation, we will use the following coupling between solutions. For $E=\mathbb{R}^{3}$ or $\mathbb{R}^{3} \times \mathbb{R}^{3}$, we denote by $C_{p}^{2}(E)$ the set of $C^{2}$ functions on $E$ of which the derivatives of order 0 to 2 have at most polynomial growth.

Proposition 10. Fix $\gamma \in(0,1]$, consider two weak solutions $\left(f_{t}\right)_{t \geq 0}$ and $\left(\tilde{f}_{t}\right)_{t \geq 0}$ to (1) such that $\int_{\mathbb{R}^{3}} e^{a|v|^{2}}\left(f_{0}+\tilde{f}_{0}\right)(\mathrm{d} v)<\infty$ for some $a>0$, and fix $R_{0} \in \mathcal{H}\left(f_{0}, \tilde{f}_{0}\right)$. There exists a family $\left(R_{t}\right)_{t \geq 0}$ of probability measures on $\mathbb{R}^{3} \times \mathbb{R}^{3}$ such that for all $t \geq 0, R_{t} \in \mathcal{H}\left(f_{t}, \tilde{f}_{t}\right)$ and for all $\psi \in C_{p}^{2}\left(\mathbb{R}^{3} \times \mathbb{R}^{3}\right)$,

$$
\begin{aligned}
\int_{\mathbb{R}^{3} \times \mathbb{R}^{3}} \psi(v, \tilde{v}) R_{t}(\mathrm{~d} v, \mathrm{~d} \tilde{v})= & \int_{\mathbb{R}^{3} \times \mathbb{R}^{3}} \psi(v, \tilde{v}) R_{0}(\mathrm{~d} v, \mathrm{~d} \tilde{v}) \\
& +\int_{0}^{t} \int_{\mathbb{R}^{3} \times \mathbb{R}^{3}} \int_{\mathbb{R}^{3} \times \mathbb{R}^{3}} \mathcal{A} \psi\left(v, v_{*}, \tilde{v}, \tilde{v}_{*}\right) R_{s}\left(\mathrm{~d} v_{*}, \mathrm{~d} \tilde{v}_{*}\right) R_{s}(\mathrm{~d} v, \mathrm{~d} \tilde{v}) \mathrm{d} s
\end{aligned}
$$

where

$$
\begin{aligned}
\mathcal{A} \psi\left(v, \tilde{v}, v_{*}, \tilde{v}_{*}\right)= & \sum_{k=1}^{3}\left[b_{k}\left(v-v_{*}\right) \partial_{v_{k}} \psi(v, \tilde{v})+b_{k}\left(\tilde{v}-\tilde{v}_{*}\right) \partial_{\tilde{v}_{k}} \psi(v, \tilde{v})\right] \\
& +\frac{1}{2} \sum_{k, \ell=1}^{3}\left[a_{k \ell}\left(v-v_{*}\right) \partial_{v_{k} v_{\ell}}^{2} \psi(v, \tilde{v})+a_{k \ell}\left(\tilde{v}-\tilde{v}_{*}\right) \partial_{\tilde{v}_{k} \tilde{v}_{\ell}}^{2} \psi(v, \tilde{v})\right] \\
& +\sum_{j, k, \ell=1}^{3} \sigma_{k j}\left(v-v_{*}\right) \sigma_{\ell j}\left(\tilde{v}-\tilde{v}_{*}\right) \partial_{v_{k} \tilde{v}_{\ell}}^{2} \psi(v, \tilde{v}) .
\end{aligned}
$$

Remark 11. Let us make the following observations.

(i) This is the key coupling of $f_{t}, \tilde{f}_{t}$ which we will use, for some well-chosen $R_{0}$, to obtain an upper bound of $\mathcal{T}_{p}\left(f_{t}, \tilde{f}_{t}\right)$ to prove Theorem 8 .

(ii) This equation has a natural probabilistic meaning: the equation governing $\left(R_{t}\right)_{t \geq 0}$ is the Kolmogorov equation for the solution $\left(V_{t}, \tilde{V}_{t}\right)_{t \geq 0}$ to the nonlinear stochastic differential equation

$$
\left\{\begin{array}{l}
V_{t}=V_{0}+\int_{0}^{t} \int_{\mathbb{R}^{3} \times \mathbb{R}^{3}} b\left(V_{s}-v_{*}\right) R_{s}\left(\mathrm{~d} v_{*}, \mathrm{~d} \tilde{v}_{*}\right) \mathrm{d} s+\int_{0}^{t} \int_{\mathbb{R}^{3} \times \mathbb{R}^{3}} \sigma\left(V_{s}-v_{*}\right) N\left(\mathrm{~d} v_{*}, \mathrm{~d} \tilde{v}_{*}, \mathrm{~d} s\right) \\
\tilde{V}_{t}=\tilde{V}_{0}+\int_{0}^{t} \int_{\mathbb{R}^{3} \times \mathbb{R}^{3}} b\left(\tilde{V}_{s}-\tilde{v}_{*}\right) R_{s}\left(\mathrm{~d} v_{*}, \mathrm{~d} \tilde{v}_{*}\right) \mathrm{d} s+\int_{0}^{t} \int_{\mathbb{R}^{3} \times \mathbb{R}^{3}} \sigma\left(\tilde{V}_{s}-\tilde{v}_{*}\right) N\left(\mathrm{~d} v_{*}, \mathrm{~d} \tilde{v}_{*}, \mathrm{~d} s\right) \\
R_{t}=\operatorname{Law}\left(V_{t}, \tilde{V}_{t}\right)
\end{array}\right.
$$

where $N=\left(N^{1}, N^{2}, N^{3}\right)$ is a $3 D$-white noise on $\mathbb{R}^{3} \times \mathbb{R}^{3} \times[0, \infty)$ with covariance measure $R_{s}\left(\mathrm{~d} v_{*}, \mathrm{~d} \tilde{v}_{*}\right) \mathrm{d}$; ; see Walsh [4]. We think of this nonlinear equation as describing the time evolution of the velocities $\left(V_{t}, \tilde{V}_{t}\right)_{t \geq 0}$ of a 'typical' pair of particles, with $V_{t} \sim f_{t}$ and $\tilde{V}_{t} \sim \tilde{f}_{t}$.

(iii) Since $R_{s} \in \mathcal{H}\left(f_{s}, \tilde{f}_{s}\right)$, we have $\int_{\mathbb{R}^{3} \times \mathbb{R}^{3}} b\left(V_{s}-v_{*}\right) R_{s}\left(\mathrm{~d} v_{*}, \mathrm{~d} \tilde{v}_{*}\right) \mathrm{d} s=\int_{\mathbb{R}^{3}} b\left(V_{s}-v_{*}\right) f_{s}\left(\mathrm{~d} v_{*}\right) \mathrm{d} s$. Similarly, $\int_{0}^{t} \int_{\mathbb{R}^{3} \times \mathbb{R}^{3}} \sigma\left(V_{s}-v_{*}\right) N\left(\mathrm{~d} v_{*}, \mathrm{~d} \tilde{v}_{*}, \mathrm{~d} s\right)=\int_{0}^{t} \int_{\mathbb{R}^{3}} \sigma\left(V_{s}-v_{*}\right) W\left(\mathrm{~d} v_{*}, \mathrm{~d} s\right)$, for some $3 D$-white noise on $\mathbb{R}^{3} \times[0, \infty)$ of covariance measure $f_{s}\left(\mathrm{~d} v_{*}\right) \mathrm{d}$ s. Hence in law, the first $S D E\left(\right.$ for $\left.\left(V_{t}\right)_{t \geq 0}\right)$ does not depend on $\left(\tilde{f}_{t}\right)_{t \geq 0}$.

(iv) The specific form of this coupling is important, rather than coupling processes using the same Brownian motion. The main idea is that we want $V_{t}$ and $\tilde{V}_{t}$ to be as close as possible. Using the white noise in this way, we isolate the effect of a coupled pair $\left(v_{*}, \tilde{v}_{*}\right)$, with $v_{*}$ as close as possible to $\tilde{v}_{*}$, in the background against our process $\left(V_{s}, \tilde{V}_{s}\right)$. It is also important that the white-noise 
covariance measure is $R_{t}\left(\mathrm{~d} v_{*}, \mathrm{~d} \tilde{v}_{*}\right) \mathrm{d} s$, with $R_{t}$ the law of $\left(V_{t}, \tilde{V}_{t}\right)$. Replacing $R_{t}$, in the covariance measure of the white noise, with any other coupling (e.g. the optimal coupling for $\mathcal{T}_{p}\left(f_{t}, \tilde{f}_{t}\right)$ ) would not allow us to use some symmetry arguments.

(v) We do not claim the uniqueness of solutions to (24); existence is sufficient for our needs.

Proof of Proposition 10. We sketch the proof, as the key points are standard for nonlinear diffusion equations and the Landau equation, see Guérin 25. We fix $k \geq 1$ and define the truncated two level coefficients $B_{k}: \mathbb{R}^{3} \times \mathbb{R}^{3} \rightarrow \mathbb{R}^{3} \times \mathbb{R}^{3}$ and $\Sigma_{k}: \mathbb{R}^{3} \times \mathbb{R}^{3} \rightarrow \mathcal{M}_{6 \times 3}(\mathbb{R})$ by

$$
B_{k}\left(\begin{array}{l}
x \\
\tilde{x}
\end{array}\right)=\left(\begin{array}{l}
b_{k}(x) \\
b_{k}(\tilde{x})
\end{array}\right) ; \quad \Sigma_{k}\left(\begin{array}{l}
x \\
\tilde{x}
\end{array}\right)=\left(\begin{array}{c}
\sigma_{k}(x) \\
\sigma_{k}(\tilde{x})
\end{array}\right),
$$

where $b_{k}(x)=-2(|x| \wedge k)^{\gamma} x$ and $\sigma_{k}(x)=(|x| \wedge k)^{\gamma / 2}|x| \Pi_{x^{\perp}}$. Proceeding as in (11) and (13), one realises that $B_{k}$ and $\Sigma_{k}$ are globally Lipschitz continuous.

Now, let $W=\left(W^{1}, W^{2}, W^{3}\right)$ be a white noise on $[0, \infty) \times(0,1)$ with covariance measure $\mathrm{d} s \mathrm{~d} \alpha$. The usual arguments for nonlinear SDEs 25 imply that there exists a process $X_{t}^{k}=\left(V_{t}^{k}, \tilde{V}_{t}^{k}\right)$ with initial distribution $X_{0}^{k} \sim R_{0}$, and a copy $Y_{t}^{k}$ defined on the probability space $((0,1), \mathcal{B}(0,1), d \alpha)$, with $\operatorname{Law}\left(X_{t}^{k}\right)=\operatorname{Law}\left(Y_{t}^{k}\right)$ and for all $t \geq 0$,

$$
X_{t}^{k}=X_{0}^{k}+\int_{0}^{t} \int_{(0,1)} B_{k}\left(X_{s}^{k}-Y_{s}^{k}(\alpha)\right) \mathrm{d} \alpha \mathrm{d} s+\int_{0}^{t} \int_{(0,1)} \Sigma_{k}\left(X_{s}^{k}-Y_{s}^{k}(\alpha)\right) W(\mathrm{~d} s, \mathrm{~d} \alpha) .
$$

For $\psi \in C_{p}^{2}\left(\mathbb{R}^{3} \times \mathbb{R}^{3}\right)$, applying Itô's formula and taking expectations, we find

$$
\begin{aligned}
\mathbb{E}\left[\psi\left(X_{t}^{k}\right)\right]= & \mathbb{E}\left[\psi\left(X_{0}^{k}\right)\right]+\int_{0}^{t} \int_{(0,1)} \mathbb{E}\left[\nabla \psi\left(X_{s}^{k}\right) \cdot B_{k}\left(X_{s}^{k}-Y_{s}^{k}(\alpha)\right)\right] \mathrm{d} \alpha \mathrm{d} s \\
& +\frac{1}{2} \sum_{i, j=1}^{6} \int_{0}^{t} \int_{(0,1)} \mathbb{E}\left[\partial_{i j} \psi\left(X_{s}^{k}\right)\left[\Sigma_{k}\left(X_{s}^{k}-Y_{s}^{k}(\alpha)\right) \Sigma_{k}^{*}\left(X_{s}^{k}-Y_{s}^{k}(\alpha)\right)\right]_{i j}\right] \mathrm{d} \alpha \mathrm{d} s .
\end{aligned}
$$

Writing $R_{t}^{k}$ for the law of $X_{t}^{k}$ (and of $Y_{t}^{k}$ ), we thus get

$$
\begin{aligned}
\int_{\mathbb{R}^{3} \times \mathbb{R}^{3}} \psi(x) R_{t}^{k}(\mathrm{~d} x)= & \int_{\mathbb{R}^{3} \times \mathbb{R}^{3}} \psi(x) R_{0}(\mathrm{~d} x)+\int_{0}^{t} \int_{\mathbb{R}^{3} \times \mathbb{R}^{3}} \int_{\mathbb{R}^{3} \times \mathbb{R}^{3}} \nabla \psi(x) \cdot B_{k}\left(x-x_{*}\right) R_{s}^{k}(\mathrm{~d} x) R_{s}^{k}\left(\mathrm{~d} x_{*}\right) \mathrm{d} s \\
& \left.+\frac{1}{2} \sum_{i, j=1}^{6} \int_{0}^{t} \int_{\mathbb{R}^{3} \times \mathbb{R}^{3}} \int_{\mathbb{R}^{3} \times \mathbb{R}^{3}} \partial_{i j} \psi(x)\left[\Sigma_{k}\left(x-x_{*}\right) \Sigma_{k}^{*}\left(x-x_{*}\right)\right]_{i j}\right] R_{s}^{k}(\mathrm{~d} x) R_{s}^{k}\left(\mathrm{~d} x_{*}\right) \mathrm{d} s .
\end{aligned}
$$

This precisely rewrites as

$$
\begin{aligned}
\int_{\mathbb{R}^{3} \times \mathbb{R}^{3}} \psi(v, \tilde{v}) R_{t}^{k}(\mathrm{~d} v, \mathrm{~d} \tilde{v})= & \int_{\mathbb{R}^{3} \times \mathbb{R}^{3}} \psi(v, \tilde{v}) R_{0}(\mathrm{~d} v, \mathrm{~d} \tilde{v}) \\
& +\int_{0}^{t} \int_{\mathbb{R}^{3} \times \mathbb{R}^{3}} \int_{\mathbb{R}^{3} \times \mathbb{R}^{3}} \mathcal{A}_{k} \psi\left(v, \tilde{v}, v_{*}, \tilde{v}_{*}\right) R_{s}^{k}(\mathrm{~d} v, \mathrm{~d} \tilde{v}) R_{s}^{k}\left(\mathrm{~d} v_{*}, \mathrm{~d} \tilde{v}_{*}\right) \mathrm{d} s,
\end{aligned}
$$

where $\mathcal{A}_{k} \psi$ is defined as $\mathcal{A} \psi$, replacing everywhere $b, \sigma$ and $a=\sigma \sigma^{*}$ by $b_{k}, \sigma_{k}$ and $a_{k}=\sigma_{k} \sigma_{k}^{*}$.

For $\psi(v, \tilde{v})=\phi(v)+\phi(\tilde{v})$, we have $\mathcal{A}_{k} \psi\left(v, \tilde{v}, v_{*}, \tilde{v}_{*}\right)=\mathcal{L}_{k} \phi\left(v, v_{*}\right)+\mathcal{L}_{k} \phi\left(\tilde{v}, \tilde{v}_{*}\right)$, where $\mathcal{L}_{k} \phi$ is defined as $\mathcal{L} \phi$, replacing $b$ and $a$ by $b_{k}$ and $a_{k}$. It is then straightforward to check that the approximate equation (25) propagates moments, uniformly in $k$, using arguments similar to those of [11, Theorem 3] or Step 1 of the proof of Proposition [ In particular, under our initial Gaussian moment assumption, all moments of $R_{t}^{k}$ are bounded, uniformly $k \geq 1$, locally uniformly in $t \geq 0$. 
It is then very classical to let $k \rightarrow \infty$ in (25), using a compactness argument, and to deduce the existence of a family of probability measures $\left(R_{t}\right)_{t \geq 0}$ solving (24) for all $\psi \in C_{p}^{2}\left(\mathbb{R}^{3} \times \mathbb{R}^{3}\right)$. See Section 6 for a similar procedure (with much less moment estimates).

Finally, we address the claim that $R_{t}$ is a coupling $R_{t} \in \mathcal{H}\left(f_{t}, \tilde{f}_{t}\right)$. Let us write $g_{t}, \tilde{g}_{t}$ for the two marginals of $R_{t}$. For any $\varphi \in C_{b}^{2}\left(\mathbb{R}^{3}\right)$, we set $\psi(v, \tilde{v})=\varphi(v)$ and observe that $\mathcal{A} \psi\left(v, \tilde{v}, v_{*}, \tilde{v}_{*}\right)=$ $\mathcal{L} \varphi\left(v, v_{*}\right)$, so that (24) tells us that

$$
\int_{\mathbb{R}^{3}} \varphi(v) g_{t}(\mathrm{~d} v)=\int_{\mathbb{R}^{3}} \varphi(v) f_{0}(\mathrm{~d} v)+\int_{0}^{t} \int_{\mathbb{R}^{3}} \mathcal{L} \varphi\left(v, v_{*}\right) g_{s}\left(\mathrm{~d} v_{*}\right) g_{s}(\mathrm{~d} v) \mathrm{d} s .
$$

In other words, $\left(g_{t}\right)_{t \geq 0}$ is a weak solution to (11) which starts at $f_{0}$. Since $f_{0}$ is assumed to have a Gaussian moment, the uniqueness result Theorem 7 applies and so $\left(g_{t}\right)_{t \geq 0}=\left(f_{t}\right)_{t \geq 0}$ as desired. The argument that $\left(\tilde{g}_{t}\right)_{t \geq 0}=\left(\tilde{f}_{t}\right)_{t \geq 0}$ is identical.

We now carefully apply the coupling operator to our cost functions.

Lemma 12. Adopt the notation of Proposition 10 and fix $p \geq 2$ and $\varepsilon \in[0,1]$, and let $c_{p, \epsilon}$ be the transport cost defined in (15). For $v, v_{*}, \tilde{v}, \tilde{v}_{*} \in \mathbb{R}^{3}$,

$$
\begin{aligned}
\mathcal{A} c_{p, \varepsilon}\left(v, v_{*}, \tilde{v}, \tilde{v}_{*}\right) \leq & k_{p, \varepsilon}^{(1)}\left(v, v_{*}, \tilde{v}, \tilde{v}_{*}\right)+k_{p, \varepsilon}^{(2)}\left(v, v_{*}, \tilde{v}, \tilde{v}_{*}\right)+k_{p, \varepsilon}^{(2)}\left(\tilde{v}, \tilde{v}_{*}, v, v_{*}\right) \\
& +k_{p, \varepsilon}^{(3)}\left(v, v_{*}, \tilde{v}, \tilde{v}_{*}\right)+k_{p, \varepsilon}^{(3)}\left(\tilde{v}, \tilde{v}_{*}, v, v_{*}\right),
\end{aligned}
$$

where, setting $x=v-v_{*}$ and $\tilde{x}=\tilde{v}-\tilde{v}_{*}$,

$$
\begin{aligned}
& k_{p, \varepsilon}^{(1)}\left(v, v_{*}, \tilde{v}, \tilde{v}_{*}\right)=\left(1+|v|^{p}+|\tilde{v}|^{p}\right) \varphi_{\varepsilon}^{\prime}\left(|v-\tilde{v}|^{2}\right)\left[2(v-\tilde{v}) \cdot(b(x)-b(\tilde{x}))+\| \sigma(x)-\sigma(\tilde{x})||^{2}\right], \\
& k_{p, \varepsilon}^{(2)}\left(v, v_{*}, \tilde{v}, \tilde{v}_{*}\right)=\varphi_{\varepsilon}\left(|v-\tilde{v}|^{2}\right)\left[p|v|^{p-2} v \cdot b(x)+\frac{p}{2}|v|^{p-2}|| \sigma(x) \|^{2}+\frac{p(p-2)}{2}|v|^{p-4}|\sigma(x) v|^{2}\right], \\
& k_{p, \varepsilon}^{(3)}\left(v, v_{*}, \tilde{v}, \tilde{v}_{*}\right)=2 p|v|^{p-2} \varphi_{\varepsilon}^{\prime}\left(|v-\tilde{v}|^{2}\right)[\sigma(x) v] \cdot[(\sigma(x)-\sigma(\tilde{x}))(v-\tilde{v})] .
\end{aligned}
$$

Proof. Fix $p \geq 2, \varepsilon \geq 0$ and let $\psi(v, \tilde{v})=c_{p, \varepsilon}(v, \tilde{v})=\left(1+|v|^{p}+|\tilde{v}|^{p}\right) \varphi_{\varepsilon}\left(|v-\tilde{v}|^{2}\right)$. We have

$$
\partial_{v_{k}} \psi(v, \tilde{v})=p|v|^{p-2} v_{k} \varphi_{\varepsilon}\left(|v-\tilde{v}|^{2}\right)+2\left(v_{k}-\tilde{v}_{k}\right)\left(1+|v|^{p}+|\tilde{v}|^{p}\right) \varphi_{\varepsilon}^{\prime}\left(|v-\tilde{v}|^{2}\right)
$$

and a symmetric expression for $\partial_{\tilde{v}_{k}} \psi(v, \tilde{v})$. Differentiating again, we find

$$
\begin{aligned}
\partial_{v_{k} v_{\ell}}^{2} \psi(v, \tilde{v})= & p|v|^{p-2} \mathbb{I}_{\{k=\ell\}} \varphi_{\varepsilon}\left(|v-\tilde{v}|^{2}\right)+p(p-2)|v|^{p-4} v_{k} v_{\ell} \varphi_{\varepsilon}\left(|v-\tilde{v}|^{2}\right) \\
& +2 p|v|^{p-2} v_{k}\left(v_{\ell}-\tilde{v}_{\ell}\right) \varphi_{\varepsilon}^{\prime}\left(|v-\tilde{v}|^{2}\right)+2 \mathbb{I}_{\{k=\ell\}}\left(1+|v|^{p}+|\tilde{v}|^{p}\right) \varphi_{\varepsilon}^{\prime}\left(|v-\tilde{v}|^{2}\right) \\
& +4\left(v_{k}-\tilde{v}_{k}\right)\left(v_{\ell}-\tilde{v}_{\ell}\right)\left(1+|v|^{p}+|\tilde{v}|^{p}\right) \varphi_{\varepsilon}^{\prime \prime}\left(|v-\tilde{v}|^{2}\right) \\
& +2 p|v|^{p-2}\left(v_{k}-\tilde{v}_{k}\right) v_{\ell} \varphi_{\varepsilon}^{\prime}\left(|v-\tilde{v}|^{2}\right)
\end{aligned}
$$

and a symmetric expression for $\partial_{\tilde{v}_{k} \tilde{v}_{\ell}}^{2} \psi(v, \tilde{v})$. Concerning the cross terms,

$$
\begin{aligned}
\partial_{v_{k} \tilde{v}_{\ell}}^{2} \psi(v, \tilde{v})= & 2 p|v|^{p-2} v_{k}\left(\tilde{v}_{\ell}-v_{\ell}\right) \varphi_{\varepsilon}^{\prime}\left(|v-\tilde{v}|^{2}\right)+2 p|\tilde{v}|^{p-2}\left(v_{k}-\tilde{v}_{k}\right) \tilde{v}_{\ell} \varphi_{\varepsilon}^{\prime}\left(|v-\tilde{v}|^{2}\right) \\
& -4\left(v_{k}-\tilde{v}_{k}\right)\left(v_{\ell}-\tilde{v}_{\ell}\right)\left(1+|v|^{p}+|\tilde{v}|^{p}\right) \varphi_{\varepsilon}^{\prime \prime}\left(|v-\tilde{v}|^{2}\right) \\
& -2 \mathbb{I}_{\{k=\ell\}}\left(1+|v|^{p}+|\tilde{v}|^{p}\right) \varphi_{\varepsilon}^{\prime}\left(|v-\tilde{v}|^{2}\right) .
\end{aligned}
$$


Let us now examine the sums in the definition of $\mathcal{A} \psi$ one by one. First,

$$
\begin{aligned}
& \sum_{k=1}^{3}\left[b_{k}\left(v-v_{*}\right) \partial_{v_{k}} \psi(v, \tilde{v})+b_{k}\left(\tilde{v}-\tilde{v}_{*}\right) \partial_{\tilde{v}_{k}} \psi(v, \tilde{v})\right] \\
& =p|v|^{p-2} v \cdot b\left(v-v_{*}\right) \varphi_{\varepsilon}\left(|v-\tilde{v}|^{2}\right) \quad\left(=A_{1}\right) \\
& +p|\tilde{v}|^{p-2} \tilde{v} \cdot b\left(\tilde{v}-\tilde{v}_{*}\right) \varphi_{\varepsilon}\left(|v-\tilde{v}|^{2}\right) \quad \quad\left(=A_{2}\right) \\
& +2\left(1+|v|^{p}+|\tilde{v}|^{p}\right)(v-\tilde{v}) \cdot\left(b\left(v-v_{*}\right)-b\left(\tilde{v}-\tilde{v}_{*}\right)\right) \varphi_{\varepsilon}^{\prime}\left(|v-\tilde{v}|^{2}\right) . \quad\left(=A_{3}\right)
\end{aligned}
$$

Next, using that for $x, y, z \in \mathbb{R}^{3}, \operatorname{Tr} a(x)=\|\sigma(x)\|^{2}$ and $\sum_{k, \ell=1}^{3} a_{k \ell}(x) y_{k} z_{\ell}=[\sigma(x) y] \cdot[\sigma(x) z]$,

$$
\begin{array}{rlrl}
\frac{1}{2} \sum_{k, \ell=1}^{3} a_{k \ell}\left(v-v_{*}\right) & \partial_{v_{k}}^{2} v_{\ell} \psi(v, \tilde{v})=\frac{p}{2}|v|^{p-2}\left\|\sigma\left(v-v_{*}\right)\right\|^{2} \varphi_{\varepsilon}\left(|v-\tilde{v}|^{2}\right) & & \left(=B_{1}\right) \\
& +\frac{p(p-2)}{2}|v|^{p-4}\left|\sigma\left(v-v_{*}\right) v\right|^{2} \varphi_{\varepsilon}\left(|v-\tilde{v}|^{2}\right) & & \left(=B_{2}\right) \\
& +2 p|v|^{p-2}\left[\sigma\left(v-v_{*}\right) v\right] \cdot\left[\sigma\left(v-v_{*}\right)(v-\tilde{v})\right] \varphi_{\varepsilon}^{\prime}\left(|v-\tilde{v}|^{2}\right) & \left(=B_{3}\right) \\
& +\left(1+|v|^{p}+|\tilde{v}|^{p}\right)\left\|\sigma\left(v-v_{*}\right)\right\|^{2} \varphi_{\varepsilon}^{\prime}\left(|v-\tilde{v}|^{2}\right) & \left(=B_{4}\right) \\
& +2\left(1+|v|^{p}+|\tilde{v}|^{p}\right)\left|\sigma\left(v-v_{*}\right)(v-\tilde{v})\right|^{2} \varphi_{\varepsilon}^{\prime \prime}\left(|v-\tilde{v}|^{2}\right) . & & \left(=B_{5}\right)
\end{array}
$$

Similarly,

$$
\begin{array}{rlrl}
\frac{1}{2} \sum_{k, \ell=1}^{3} a_{k \ell}\left(\tilde{v}-\tilde{v}_{*}\right) & \partial_{\tilde{v}_{k}}^{2} \tilde{v}_{\ell} \psi(v, \tilde{v})=\frac{p}{2}|\tilde{v}|^{p-2}\left\|\sigma\left(\tilde{v}-\tilde{v}_{*}\right)\right\|^{2} \varphi_{\varepsilon}\left(|v-\tilde{v}|^{2}\right) & & \left(=C_{1}\right) \\
& +\frac{p(p-2)}{2}|\tilde{v}|^{p-4}\left|\sigma\left(\tilde{v}-\tilde{v}_{*}\right) \tilde{v}\right|^{2} \varphi_{\varepsilon}\left(|v-\tilde{v}|^{2}\right) & \left(=C_{2}\right) \\
& +2 p|\tilde{v}|^{p-2}\left[\sigma\left(\tilde{v}-\tilde{v}_{*}\right) \tilde{v}\right] \cdot\left[\sigma\left(\tilde{v}-\tilde{v}_{*}\right)(\tilde{v}-v)\right] \varphi_{\varepsilon}^{\prime}\left(|v-\tilde{v}|^{2}\right) & \left(=C_{3}\right) \\
& +\left(1+|v|^{p}+|\tilde{v}|^{p}\right)\left\|\sigma\left(\tilde{v}-\tilde{v}_{*}\right)\right\|^{2} \varphi_{\varepsilon}^{\prime}\left(|v-\tilde{v}|^{2}\right) & \left(=C_{4}\right) \\
& +2\left(1+|v|^{p}+|\tilde{v}|^{p}\right)\left|\sigma\left(\tilde{v}-\tilde{v}_{*}\right)(\tilde{v}-v)\right|^{2} \varphi_{\varepsilon}^{\prime \prime}\left(|v-\tilde{v}|^{2}\right) . & & \left(=C_{5}\right)
\end{array}
$$

Finally, we look at the cross-terms:

$$
\begin{array}{rlr} 
& \sum_{j, k, \ell=1}^{3} \sigma_{k j}\left(v-v_{*}\right) \sigma_{\ell j}\left(\tilde{v}-\tilde{v}_{*}\right) \partial_{v_{k} \tilde{v}_{\ell}}^{2} \psi(v, \tilde{v}) & \\
= & -2 p|v|^{p-2}\left[\sigma\left(v-v_{*}\right) v\right] \cdot\left[\sigma\left(\tilde{v}-\tilde{v}_{*}\right)(v-\tilde{v})\right] \varphi_{\varepsilon}^{\prime}\left(|v-\tilde{v}|^{2}\right) & \left(=D_{1}\right) \\
& +2 p|\tilde{v}|^{p-2}\left[\sigma\left(v-v_{*}\right)(v-\tilde{v})\right] \cdot\left[\sigma\left(\tilde{v}-\tilde{v}_{*}\right) \tilde{v}\right] \varphi_{\varepsilon}^{\prime}\left(|v-\tilde{v}|^{2}\right) & \left(=D_{2}\right) \\
& -4\left(1+|v|^{p}+|\tilde{v}|^{p}\right)\left[\sigma\left(v-v_{*}\right)(v-\tilde{v})\right] \cdot\left[\sigma\left(\tilde{v}-\tilde{v}_{*}\right)(v-\tilde{v})\right] \varphi_{\varepsilon}^{\prime \prime}\left(|v-\tilde{v}|^{2}\right) & \left(=D_{3}\right) \\
& -2\left(1+|v|^{p}+|\tilde{v}|^{p}\right)\left\langle\left\langle\sigma\left(v-v_{*}\right), \sigma\left(\tilde{v}-\tilde{v}_{*}\right)\right\rangle\right\rangle \varphi_{\varepsilon}^{\prime}\left(|v-\tilde{v}|^{2}\right) . &
\end{array}
$$


Recalling the notation $x=v-v_{*}$ and $\tilde{x}=\tilde{v}-\tilde{v}_{*}$, we find that

$$
\begin{aligned}
A_{3}+B_{4}+C_{4}+D_{4} & =k_{p, \varepsilon}^{(1)}\left(v, v_{*}, \tilde{v}, \tilde{v}_{*}\right), \\
A_{1}+B_{1}+B_{2} & =k_{p, \varepsilon}^{(2)}\left(v, v_{*}, \tilde{v}, \tilde{v}_{*}\right), \\
A_{2}+C_{1}+C_{2} & =k_{p, \varepsilon}^{(2)}\left(\tilde{v}, \tilde{v}_{*}, v, v_{*}\right), \\
B_{3}+D_{1} & =k_{p, \varepsilon}^{(3)}\left(v, v_{*}, \tilde{v}, \tilde{v}_{*}\right), \\
C_{3}+D_{2} & =k_{p, \varepsilon}^{(3)}\left(\tilde{v}, \tilde{v}_{*}, v, v_{*}\right),
\end{aligned}
$$

and finally that

$$
B_{5}+C_{5}+D_{3}=2\left(1+|v|^{p}+|\tilde{v}|^{p}\right)|(\sigma(x)-\sigma(\tilde{x}))(v-\tilde{v})|^{2} \varphi_{\varepsilon}^{\prime \prime}\left(|v-\tilde{v}|^{2}\right) \leq 0
$$

since $\varphi_{\varepsilon}^{\prime \prime}$ is nonpositive, see (16).

We finally state the following central inequality.

Lemma 13. There is a constant $C$, depending only on $p \geq 2$ and $\gamma \in(0,1]$, such that for all $\varepsilon \in(0,1]$, all $v, v_{*}, \tilde{v}, \tilde{v}_{*} \in \mathbb{R}^{3}$,

$$
\begin{aligned}
\mathcal{A} c_{p, \varepsilon}\left(v, v_{*}, \tilde{v}, \tilde{v}_{*}\right) \leq & {[2-p] c_{p+\gamma, \varepsilon}(v, \tilde{v}) } \\
& +C \sqrt{\varepsilon}\left(1+\left|v_{*}\right|^{p}+\left|\tilde{v}_{*}\right|^{p}\right) c_{p+\gamma, \varepsilon}(v, \tilde{v}) \\
& +C \sqrt{\varepsilon}\left(1+|v|^{p}+|\tilde{v}|^{p}\right) c_{p+\gamma, \varepsilon}\left(v_{*}, \tilde{v}_{*}\right) \\
& +\frac{C}{\sqrt{\varepsilon}}\left(1+\left|v_{*}\right|^{p+\gamma}+\left|\tilde{v}_{*}\right|^{p+\gamma}\right) c_{p, \varepsilon}(v, \tilde{v}) \\
& +\frac{C}{\sqrt{\varepsilon}}\left(1+|v|^{p+\gamma}+|\tilde{v}|^{p+\gamma}\right) c_{p, \varepsilon}\left(v_{*}, \tilde{v}_{*}\right) .
\end{aligned}
$$

Let us now highlight the main features of this bound, which motivate our strategy. The last two lines are amenable to a Grönwall-type estimate, provided $\int_{0}^{T} m_{p+\gamma}\left(f_{s}+\tilde{f}_{s}\right) \mathrm{d} s<\infty$, but this is prevented by the appearance of $c_{p+\gamma, \varepsilon}$ in the earlier terms; in [15], analagous terms are handled using an exponential moment estimate. The key observation is that, by choosing $p>2$, the first line gives a negative multiple of this 'bad' term, which can absorb the second and third lines if $\epsilon>0$ is small enough (and if we know that $\sup _{[0, T]} m_{p}\left(f_{s}+\tilde{f}_{s}\right)<\infty$ ), allowing us to use a Grönwall estimate.

Let us mention that a rather direct computation, with $\varepsilon=0$, i.e. with the cost $c_{p, 0}(v, \tilde{v})=$ $\left(1+|v|^{p}+|\tilde{v}|^{p}\right)|v-\tilde{v}|^{2}$, relying on the simple estimates (11), (13) and (14), shows that

$$
\begin{aligned}
\mathcal{A} c_{p, 0}\left(v, v_{*}, \tilde{v}, \tilde{v}_{*}\right) \leq & {[32-p] c_{p+\gamma, 0}(v, \tilde{v}) } \\
& +C\left(1+\left|v_{*}\right|^{p+\gamma}+\left|\tilde{v}_{*}\right|^{p+\gamma}\right) c_{p, 0}(v, \tilde{v})+C\left(1+|v|^{p+\gamma}+|\tilde{v}|^{p+\gamma}\right) c_{p, 0}\left(v_{*}, \tilde{v}_{*}\right) .
\end{aligned}
$$

Choosing $p=32$, the first term is nonpositive, and this would lead to a stability result for the cost $\mathcal{T}_{32,0}$, for initial conditions in $\mathcal{P}_{34}\left(\mathbb{R}^{3}\right)$, since $\mathcal{T}_{32,0}$ requires some moments of order 34 to be well-defined.

The proof of Lemma 13 is much more complicated; we have to be very careful and to use many cancelations to replace $[32-p]$ by $[2-p]$. Moreover, we have to deal with $c_{p, \varepsilon}$ with $\varepsilon>0$ instead of $c_{p, 0}$, because $\mathcal{T}_{p, 0}$ requires moments of order $p+2$ to be well-defined. All this is crucial to obtain a stability result in $\mathcal{P}_{p}\left(\mathbb{R}^{3}\right)$, for any $p>2$. Since the proof is rather lengthy, it is deferred to Section 8 for the ease of readability. 


\section{Stability}

We now give the proof of our stability estimate. We first deal with the case when the initial data have a finite Gaussian moment, and then carefully relax this assumption.

Lemma 14. Fix $\gamma \in(0,1]$ and let $\left(f_{t}\right)_{t \geq 0},\left(\tilde{f}_{t}\right)_{t \geq 0}$ be weak solutions to (11) with initial moments $\int_{\mathbb{R}^{3}} e^{a|v|^{2}}\left(f_{0}+\tilde{f}_{0}\right)(\mathrm{d} v)<\infty$ for some $a>0$. Then the stability estimate (17) holds true.

Proof. We fix $p>2$, consider $\varepsilon \in(0,1]$ to be chosen later and introduce $R_{0} \in \mathcal{H}\left(f_{0}, \tilde{f}_{0}\right)$ such that

$$
\mathcal{T}_{p, \varepsilon}\left(f_{0}, \tilde{f}_{0}\right)=\int_{\mathbb{R}^{3} \times \mathbb{R}^{3}} c_{p, \varepsilon}(v, \tilde{v}) R_{0}(\mathrm{~d} v, \mathrm{~d} \tilde{v}) .
$$

Note that $R_{0}$ depends on $\varepsilon$, but this is not an issue. We then introduce $\left(R_{t}\right)_{t>0}$ as in Proposition 10. which is licit thanks to our initial Gaussian moment condition. We know that for each $t \geq 0$, $R_{t} \in \mathcal{H}\left(f_{t}, \tilde{f}_{t}\right)$, from which we conclude that

$$
u_{\varepsilon}(t)=\int_{\mathbb{R}^{3} \times \mathbb{R}^{3}} c_{p, \varepsilon}(v, \tilde{v}) R_{t}(\mathrm{~d} v, \mathrm{~d} \tilde{v}) \geq \mathcal{T}_{p, \varepsilon}\left(f_{t}, \tilde{f}_{t}\right) .
$$

By Proposition 10, and since $u_{\varepsilon}(0)=\mathcal{T}_{p, \varepsilon}\left(f_{0}, \tilde{f}_{0}\right)$, it holds that for all $t \geq 0$,

$$
u_{\varepsilon}(t)=\mathcal{T}_{p, \varepsilon}\left(f_{0}, \tilde{f}_{0}\right)+\int_{0}^{t} \int_{\mathbb{R}^{3} \times \mathbb{R}^{3}} \int_{\mathbb{R}^{3} \times \mathbb{R}^{3}} \mathcal{A} c_{p, \varepsilon}\left(v, v_{*}, \tilde{v}, \tilde{v}_{*}\right) R_{s}\left(\mathrm{~d} v_{*}, \mathrm{~d} \tilde{v}_{*}\right) R_{s}(\mathrm{~d} v, \mathrm{~d} \tilde{v}) \mathrm{d} s .
$$

Using next Lemma 13 and a symmetry argument, we find that

$$
u_{\varepsilon}(t) \leq \mathcal{T}_{p, \varepsilon}\left(f_{0}, \tilde{f}_{0}\right)+\int_{0}^{t}\left(I_{1, \varepsilon}(s)+I_{2, \varepsilon}(s)+I_{3, \varepsilon}(s)\right) \mathrm{d} s,
$$

where, for some constant $C>0$ depending only on $p$ and $\gamma$,

$$
\begin{aligned}
& I_{1, \varepsilon}(s)=[2-p] \int_{\mathbb{R}^{3} \times \mathbb{R}^{3}} c_{p+\gamma, \varepsilon}(v, \tilde{v}) R_{s}(\mathrm{~d} v, \mathrm{~d} \tilde{v}), \\
& I_{2, \varepsilon}(s)=C \sqrt{\varepsilon} \int_{\mathbb{R}^{3} \times \mathbb{R}^{3}} \int_{\mathbb{R}^{3} \times \mathbb{R}^{3}}\left(1+\left|v_{*}\right|^{p}+\left|\tilde{v}_{*}\right|^{p}\right) c_{p+\gamma, \varepsilon}(v, \tilde{v}) R_{s}\left(\mathrm{~d} v_{*}, \mathrm{~d} \tilde{v}_{*}\right) R_{s}(\mathrm{~d} v, \mathrm{~d} \tilde{v}), \\
& I_{3, \varepsilon}(s)=\frac{C}{\sqrt{\varepsilon}} \int_{\mathbb{R}^{3} \times \mathbb{R}^{3}} \int_{\mathbb{R}^{3} \times \mathbb{R}^{3}}\left(1+\left|v_{*}\right|^{p+\gamma}+\left|\tilde{v}_{*}\right|^{p+\gamma}\right) c_{p, \varepsilon}(v, \tilde{v}) R_{s}\left(\mathrm{~d} v_{*}, \mathrm{~d} \tilde{v}_{*}\right) R_{s}(\mathrm{~d} v, \mathrm{~d} \tilde{v}) .
\end{aligned}
$$

Using that $R_{s} \in \mathcal{H}\left(f_{s}, \tilde{f}_{s}\right)$, we conclude that

$$
\begin{aligned}
& I_{2, \varepsilon}(s) \leq C \sqrt{\varepsilon}\left(1+m_{p}\left(f_{s}+\tilde{f}_{s}\right)\right) \int_{\mathbb{R}^{3} \times \mathbb{R}^{3}} c_{p+\gamma, \varepsilon}(v, \tilde{v}) R_{s}(\mathrm{~d} v, \mathrm{~d} \tilde{v}), \\
& I_{3, \varepsilon}(s) \leq \frac{C}{\sqrt{\varepsilon}}\left(1+m_{p+\gamma}\left(f_{s}+\tilde{f}_{s}\right)\right) u_{\varepsilon}(s) .
\end{aligned}
$$

We now fix $t>0$ and work on $[0, t]$. Setting $m_{p, \infty}([0, t])=\sup _{s \in[0, t]} m_{p}\left(f_{s}+\tilde{f}_{s}\right)$ and choosing

$$
\varepsilon=\left[\frac{p-2}{p-2+C\left(1+m_{p, \infty}([0, t])\right)}\right]^{2},
$$

so that $\varepsilon \in(0,1]$ and $2-p+C \sqrt{\varepsilon}\left(1+m_{p}\left(f_{s}+\tilde{f}_{s}\right)\right) \leq 0$ for all $s \in[0, t]$, we conclude that $I_{1, \varepsilon}(s)+I_{2, \varepsilon}(s) \leq 0$ for all $s \in[0, t]$, whence

$$
u_{\varepsilon}(r) \leq \mathcal{T}_{p, \varepsilon}\left(f_{0}, \tilde{f}_{0}\right)+\frac{C}{\sqrt{\varepsilon}} \int_{0}^{r}\left(1+m_{p+\gamma}\left(f_{s}+\tilde{f}_{s}\right)\right) u_{\varepsilon}(s) \mathrm{d} s
$$


for all $r \in[0, t]$. The Grönwall lemma then tells us that

$$
\mathcal{T}_{p, \varepsilon}\left(f_{t}, \tilde{f}_{t}\right) \leq u_{\varepsilon}(t) \leq \mathcal{T}_{p, \varepsilon}\left(f_{0}, \tilde{f}_{0}\right) \exp \left(\frac{C}{\sqrt{\varepsilon}} \int_{0}^{t}\left(1+m_{p+\gamma}\left(f_{s}+\tilde{f}_{s}\right)\right) \mathrm{d} s\right) .
$$

Using finally that $\mathcal{T}_{p}=\mathcal{T}_{p, 1}$ and that $c_{p, 1} \leq c_{p, \varepsilon} \leq \varepsilon^{-1} c_{p, 1}$, we deduce that

$$
\mathcal{T}_{p}\left(f_{t}, \tilde{f}_{t}\right) \leq \mathcal{T}_{p, \varepsilon}\left(f_{t}, \tilde{f}_{t}\right) \quad \text { and } \quad \mathcal{T}_{p, \varepsilon}\left(f_{0}, \tilde{f}_{0}\right) \leq \frac{1}{\varepsilon} \mathcal{T}_{p}\left(f_{0}, \tilde{f}_{0}\right) .
$$

We thus end with

$$
\mathcal{T}_{p}\left(f_{t}, \tilde{f}_{t}\right) \leq \frac{1}{\varepsilon} \mathcal{T}_{p}\left(f_{0}, \tilde{f}_{0}\right) \exp \left(\frac{C}{\sqrt{\varepsilon}} \int_{0}^{t}\left(1+m_{p+\gamma}\left(f_{s}+\tilde{f}_{s}\right)\right) \mathrm{d} s\right) .
$$

Recalling our choice for $\varepsilon$ and allowing the value of $C$, still depending only on $p$ and $\gamma$, to change from line to line, we find that

$$
\begin{aligned}
\mathcal{T}_{p}\left(f_{t}, \tilde{f}_{t}\right) & \leq C\left(1+m_{p, \infty}([0, t])\right)^{2} \mathcal{T}_{p}\left(f_{0}, \tilde{f}_{0}\right) \exp \left(C\left[1+m_{p, \infty}([0, t])\right] \int_{0}^{t}\left(1+m_{p+\gamma}\left(f_{s}+\tilde{f}_{s}\right)\right) \mathrm{d} s\right) \\
& \leq \mathcal{T}_{p}\left(f_{0}, \tilde{f}_{0}\right) \exp \left(C\left[1+m_{p, \infty}([0, t])\right]\left[1+\int_{0}^{t}\left(1+m_{p+\gamma}\left(f_{s}+\tilde{f}_{s}\right)\right) \mathrm{d} s\right]\right)
\end{aligned}
$$

which was our goal.

In order to relax the initial Gaussian moment condition, we will use the following convergence.

Lemma 15. Fix $\gamma \in(0,1]$ and $p>2$. Let $\left(f_{t}\right)_{t \geq 0}$ be a weak solution to (11), with initial moment $m_{p}\left(f_{0}\right)<\infty$. Then $\mathcal{T}_{p}\left(f_{t}, f_{0}\right) \rightarrow 0$ as $t \rightarrow 0$.

Proof. First, thanks to the density of $C_{b}^{2}\left(\mathbb{R}^{3}\right)$ in $C_{b}\left(\mathbb{R}^{3}\right)$, we deduce from (3) that $f_{t} \rightarrow f_{0}$ weakly. It classically follows that $\lim _{t \rightarrow 0} d\left(f_{t}, f_{0}\right)=0$, where $d$ is the following distance that classicaly metrises weak convergence on probability measures:

$$
d(f, g)=\inf \left\{\int_{\mathbb{R}^{3} \times \mathbb{R}^{3}}(1 \wedge|v-w|) S(\mathrm{~d} v, \mathrm{~d} w): S \in \mathcal{H}(f, g)\right\} .
$$

Moreover, for each $t \geq 0$, there exists a coupling $S_{t} \in \mathcal{H}\left(f_{t}, f_{0}\right)$ attaing the minimum $d\left(f_{t}, f_{0}\right)=$ $\int_{\mathbb{R}^{3} \times \mathbb{R}^{3}}(1 \wedge|v-w|) S_{t}(\mathrm{~d} v, \mathrm{~d} w)$. Now, fix $\epsilon>0$; by Lemma 9, there exist $M<\infty$ and $t_{0}>0$ such that

$$
\int_{\mathbb{R}^{3}}\left(1+|v|^{p}\right) \mathbb{I}_{\{|v|>M\}} f_{t}(\mathrm{~d} v)<\epsilon \quad \text { for all } t \in\left[0, t_{0}\right] .
$$

Since now $c_{p, 1}(v, w) \leq\left(1+|v|^{p}+|w|^{p}\right)(|v-w| \wedge 1) \leq\left(1+|v|^{p}\right)(|v-w| \wedge 1)+\left(1+|w|^{p}\right)(|v-w| \wedge 1)$ and since $\mathcal{T}_{p}=\mathcal{T}_{p, 1}$, we have

$$
\begin{aligned}
\mathcal{T}_{p}\left(f_{t}, f_{0}\right) \leq & \int_{\mathbb{R}^{3} \times \mathbb{R}^{3}} c_{p, 1}(v, w) S_{t}(\mathrm{~d} v, \mathrm{~d} w) \\
\leq & \left(1+M^{p}\right) d\left(f_{t}, f_{0}\right)+\int_{\mathbb{R}^{3} \times \mathbb{R}^{3}}\left(1+|v|^{p}\right) \mathbb{I}_{\{|v|>M\}} S_{t}(\mathrm{~d} v, \mathrm{~d} w) \\
& +\left(1+M^{p}\right) d\left(f_{t}, f_{0}\right)+\int_{\mathbb{R}^{3} \times \mathbb{R}^{3}}\left(1+|w|^{p}\right) \mathbb{I}_{\{|w|>M\}} S_{t}(\mathrm{~d} v, \mathrm{~d} w) \\
= & 2\left(1+M^{p}\right) d\left(f_{t}, f_{0}\right)+\int_{\mathbb{R}^{3}}\left(1+|v|^{p}\right) \mathbb{I}_{\{|v|>M\}} f_{t}(\mathrm{~d} v)+\int_{\mathbb{R}^{3}}\left(1+|w|^{p}\right) \mathbb{I}_{\{|w|>M\}} f_{0}(\mathrm{~d} w),
\end{aligned}
$$

the last equality using that $S_{t} \in \mathcal{H}\left(f_{t}, f_{0}\right)$. We conclude that for all $t \in\left[0, t_{0}\right]$,

$$
\mathcal{T}_{p}\left(f_{t}, f_{0}\right) \leq 2\left(1+M^{p}\right) d\left(f_{t}, f_{0}\right)+2 \varepsilon,
$$


whence $\lim \sup _{t \rightarrow 0} \mathcal{T}_{p}\left(f_{t}, f_{0}\right) \leq 2 \varepsilon$ and we are done, as $\epsilon>0$ was arbitrary.

We are now ready to remove the additional assumptions and prove the full stability statement.

Proof of Theorem [8. We fix $\gamma \in(0,1], p>2$ and we consider two weak solutions $\left(f_{t}\right)_{t \geq 0}$ and $\left(\tilde{f}_{t}\right)_{t \geq 0}$ to (11) such that $m_{p}\left(f_{0}+\tilde{f}_{0}\right)<\infty$.

Fix $t>0$ and let $0<s \leq t$; thanks to Proposition 6 we have $\int_{\mathbb{R}^{3}} e^{a|v|^{2}}\left(f_{s}+\tilde{f}_{s}\right)(\mathrm{d} v)<\infty$ for some $a>0$. Lemma 14 therefore applies to $\left(f_{u}\right)_{u \geq s},\left(\tilde{f}_{u}\right)_{u \geq s}$, so that, setting $m_{p, \infty}([s, t])=$ $\sup _{r \in[s, t]} m_{p}\left(f_{r}+\tilde{f}_{r}\right)$,

$$
\begin{aligned}
\mathcal{T}_{p}\left(f_{t}, \tilde{f}_{t}\right) & \leq \mathcal{T}_{p}\left(f_{s}, \tilde{f}_{s}\right) \exp \left(C\left[1+m_{p, \infty}([s, t])\right]\left[1+\int_{s}^{t}\left(1+m_{p+\gamma}\left(f_{u}+\tilde{f}_{u}\right)\right) \mathrm{d} u\right]\right) \\
& \leq \mathcal{T}_{p}\left(f_{s}, \tilde{f}_{s}\right) \exp \left(C\left[1+m_{p, \infty}([0, t])\right]\left[1+\int_{0}^{t}\left(1+m_{p+\gamma}\left(f_{u}+\tilde{f}_{u}\right)\right) \mathrm{d} u\right]\right) .
\end{aligned}
$$

Recalling the relaxed triangle inequality (18), we have, for some constant $C$ depending only on $p$,

$$
\mathcal{T}_{p}\left(f_{s}, \tilde{f}_{s}\right) \leq C\left[\mathcal{T}_{p}\left(f_{s}, f_{0}\right)+\mathcal{T}_{p}\left(f_{0}, \tilde{f}_{0}\right)+\mathcal{T}_{p}\left(\tilde{f}_{0}, \tilde{f}_{s}\right)\right]
$$

and as $s \rightarrow 0$, the first and third terms converge to 0 by Lemma [15, so

$$
\limsup _{s \rightarrow 0} \mathcal{T}_{p}\left(f_{s}, \tilde{f}_{s}\right) \leq C \mathcal{T}_{p}\left(f_{0}, \tilde{f}_{0}\right) .
$$

We thus can take $s \downarrow 0$ in (27) to obtain the desired result.

\section{EXISTENCE}

Proof of Theorem [3. Let us start from $f_{0} \in \mathcal{P}_{2}$. By the de La Vallée Poussin theorem, there exists a $C^{2}$-function $h:[0, \infty) \rightarrow[0, \infty)$ such that $h^{\prime \prime} \geq 0, h^{\prime}(\infty)=\infty$ and

$$
\int_{\mathbb{R}^{3}} h\left(|v|^{2}\right) f_{0}(\mathrm{~d} v)<\infty .
$$

We can also impose that $h^{\prime \prime} \leq 1$ and that $h^{\prime}(0)=1$.

Step 1. We consider $n_{0} \geq 1$ such that for all $n \geq n_{0}, \alpha_{n}=\int_{\mathbb{R}^{3}} \mathbb{I}_{\{|v| \leq n\}} f_{0}(\mathrm{~d} v) \geq 1 / 2$ and set, for $n \geq n_{0}$,

$$
f_{0}^{n}(\mathrm{~d} v)=\alpha_{n}^{-1} \mathbb{I}_{\{|v| \leq n\}} f_{0}(\mathrm{~d} v) \in \mathcal{P}\left(\mathbb{R}^{3}\right) .
$$

Since $f_{0}^{n}$ is compactly supported, it has all moments finite and there exists a weak solution $\left(f_{t}^{n}\right)_{t \geq 0}$ to (11) starting at $f_{0}^{n}$ by Theorem 2. Of course, $f_{0}^{n}$ converges weakly to $f_{0}$ as $n \rightarrow \infty$.

Step 2. We now show that for all $T>0$, there is a finite constant $K_{T}$ such that for all $n \geq n_{0}$,

$$
\sup _{t \in[0, T]} \int_{\mathbb{R}^{3}} h\left(|v|^{2}\right) f_{t}^{n}(\mathrm{~d} v)+\int_{0}^{T} \int_{\mathbb{R}^{3}}|v|^{2+\gamma} h^{\prime}\left(|v|^{2}\right) f_{t}^{n}(\mathrm{~d} v) \mathrm{d} t \leq K_{T} .
$$

By Theorem 2, all polynomial moments of $f_{t}^{n}$ are bounded, uniformly in $t \geq 0$ (but not necessarily in $n)$. We can therefore apply (3) to the function $\varphi(v)=h\left(|v|^{2}\right)$ : arguing as in (20),

$$
\partial_{k} \varphi(v)=2 v_{k} h^{\prime}\left(|v|^{2}\right) ; \quad \partial_{k \ell}^{2} \varphi\left(|v|^{2}\right)=2 h^{\prime}\left(|v|^{2}\right) \mathbb{1}_{\{k=\ell\}}+4 v_{k} v_{l} h^{\prime \prime}\left(|v|^{2}\right)
$$

and so, setting $x=v-v_{*}$ as usual,

$$
\mathcal{L} \varphi\left(v, v_{*}\right)=h^{\prime}\left(|v|^{2}\right)\left[2 v \cdot b(x)+\|\sigma(x)\|^{2}\right]+2|\sigma(x) v|^{2} h^{\prime \prime}\left(|v|^{2}\right) .
$$

Recalling (14) and that $0 \leq h^{\prime \prime} \leq 1$, the last term is bounded by

$$
2|\sigma(x) v|^{2} h^{\prime \prime}\left(|v|^{2}\right) \leq C|x|^{\gamma}|v|^{2}\left|v_{*}\right|^{2} \leq C\left(|v|^{2+\gamma}\left|v_{*}\right|^{2}+|v|^{2}\left|v_{*}\right|^{2+\gamma}\right) .
$$


Meanwhile, since $b(x)=-2|x|^{\gamma} x$ and $\|\left.\sigma(x)\right|^{2}=2|x|^{\gamma+2}$, the first term is

$$
\begin{aligned}
h^{\prime}\left(|v|^{2}\right) & {\left[2 v \cdot b(x)+\|\sigma(x)\|^{2}\right]=2 h^{\prime}\left(|v|^{2}\right)\left[-|x|^{\gamma}|v|^{2}+|x|^{\gamma}\left|v_{*}\right|^{2}\right] } \\
& \leq-2 h^{\prime}\left(|v|^{2}\right)|v|^{2+\gamma}+2 h^{\prime}\left(|v|^{2}\right)\left|v_{*}\right|^{\gamma}|v|^{2}+2 h^{\prime}\left(|v|^{2}\right)|v|^{\gamma}\left|v_{*}\right|^{2}+2 h^{\prime}\left(|v|^{2}\right)\left|v_{*}\right|^{2+\gamma} \\
& \leq-h^{\prime}\left(|v|^{2}\right)|v|^{2+\gamma}+C\left(1+|v|^{2}\right)\left|v_{*}\right|^{\gamma+2} .
\end{aligned}
$$

We used that $|x|^{\gamma} \geq|v|^{\gamma}-\left|v_{*}\right|^{\gamma}$, that $|x|^{\gamma} \leq|v|^{\gamma}-\left|v_{*}\right|^{\gamma}$ and, for the last inequality, that there is $C>0$ such that $\left|v_{*}\right|^{\gamma}|v|^{2}+|v|^{\gamma}\left|v_{*}\right|^{2} \leq \frac{1}{2}|v|^{2+\gamma}+C\left|v_{*}\right|^{2+\gamma}$ and that $h^{\prime}(r) \leq 1+r$. All in all,

$$
\mathcal{L} \varphi\left(v, v_{*}\right) \leq-h^{\prime}\left(|v|^{2}\right)|v|^{2+\gamma}+C\left(1+|v|^{2}\right)\left|v_{*}\right|^{\gamma+2}+C\left(1+\left|v_{*}\right|^{2}\right)|v|^{\gamma+2}
$$

We thus find, by (3), recalling that $m_{2}\left(f_{t}^{n}\right)=m_{2}\left(f_{0}^{n}\right)$, that

$$
\begin{aligned}
& \int_{\mathbb{R}^{3}} h\left(|v|^{2}\right) f_{t}^{n}(\mathrm{~d} v)+\int_{0}^{t} \int_{\mathbb{R}^{3}} h^{\prime}\left(|v|^{2}\right)|v|^{2+\gamma} f_{s}^{n}(\mathrm{~d} v) \mathrm{d} s \\
\leq & \int_{\mathbb{R}^{3}} h\left(|v|^{2}\right) f_{0}^{n}(\mathrm{~d} v)+2 C\left(1+m_{2}\left(f_{0}^{n}\right)\right) \int_{0}^{t} \int_{\mathbb{R}^{3}}|v|^{2+\gamma} f_{s}^{n}(\mathrm{~d} v) \mathrm{d} s \\
\leq & 2 \int_{\mathbb{R}^{3}} h\left(|v|^{2}\right) f_{0}(\mathrm{~d} v)+2 C\left(1+2 m_{2}\left(f_{0}\right)\right) \int_{0}^{t} \int_{\mathbb{R}^{3}}|v|^{2+\gamma} f_{s}^{n}(\mathrm{~d} v) \mathrm{d} s
\end{aligned}
$$

since $f_{0}^{n} \leq 2 f_{0}$. But since $h^{\prime}(\infty)=\infty$, there is a constant $\kappa$ (depending on $m_{2}\left(f_{0}\right)$ ) such that $2 C\left(1+2 m_{2}\left(f_{0}\right)\right)|v|^{2+\gamma} \leq \frac{1}{2} h^{\prime}\left(|v|^{2}\right)|v|^{2+\gamma}+\kappa$ for all $v \in \mathbb{R}^{3}$. We finally get

$$
\int_{\mathbb{R}^{3}} h\left(|v|^{2}\right) f_{t}^{n}(\mathrm{~d} v)+\frac{1}{2} \int_{0}^{t} \int_{\mathbb{R}^{3}} h^{\prime}\left(|v|^{2}\right)|v|^{2+\gamma} f_{s}^{n}(\mathrm{~d} v) \mathrm{d} s \leq 2 \int_{\mathbb{R}^{3}} h\left(|v|^{2}\right) f_{0}(\mathrm{~d} v)+\kappa t
$$

and this completes the step.

Step 3. Here we show that the family $\left(\left(f_{t}^{n}\right)_{t \geq 0}\right)_{n \geq n_{0}}$ is relatively compact in $C\left([0, \infty), \mathcal{P}\left(\mathbb{R}^{3}\right)\right)$, where $\mathcal{P}\left(\mathbb{R}^{3}\right)$ is endowed with the usual weak convergence. This last convergence can be metrised by the distance on $\mathcal{P}\left(\mathbb{R}^{3}\right)$ :

$$
\delta(f, g)=\sup _{\varphi \in C_{b, 1}^{2}}\left|\int_{\mathbb{R}^{3}} \varphi(v)(f-g)(\mathrm{d} v)\right|
$$

where $C_{b, 1}^{2}$ is the set of $C^{2}$ functions on $\mathbb{R}^{3}$ such that $\|\varphi\|_{\infty}+\|\nabla \varphi\|_{\infty}+\left\|\nabla^{2} \varphi\right\|_{\infty} \leq 1$. By the Arzelà-Ascoli theorem, it suffices to check that

(a) for all $t \geq 0$, the family $\left(f_{t}^{n}\right)_{n \geq n_{0}}$ is relatively compact in $\mathcal{P}\left(\mathbb{R}^{3}\right)$ and

(b) for all $T>0, \lim _{\varepsilon \rightarrow 0} \sup _{n \geq n_{0}} \sup _{s, t \in[0, T],|t-s| \leq \varepsilon} \delta\left(f_{t}^{n}, f_{s}^{n}\right)=0$.

Point (a) is obvious, since for all $t \geq 0$, all $n \geq n_{0}, m_{2}\left(f_{t}^{n}\right) \leq 2 m_{2}\left(f_{0}\right)$ and since the set $\left\{f \in \mathcal{P}\left(\mathbb{R}^{3}\right): m_{2}(f) \leq a\right\}$ is compact for any $a>0$. Concerning point (b), we recall that there is a constant $C$ such that for all $\varphi \in C_{b, 1}^{2},\left|\mathcal{L} \varphi\left(v, v_{*}\right)\right| \leq C\left(1+|v|^{\gamma+2}+\left|v_{*}\right|^{\gamma+2}\right)$. We thus deduce from (3) that for all $t \geq s \geq 0$, all $n \geq n_{0}$,

$\delta\left(f_{t}^{n}, f_{s}^{n}\right) \leq C \int_{s}^{t} \int_{\mathbb{R}^{3} \times \mathbb{R}^{3}}\left(1+|v|^{\gamma+2}+\left|v_{*}\right|^{\gamma+2}\right) f_{s}^{n}\left(\mathrm{~d} v_{*}\right) f_{s}^{n}(\mathrm{~d} v) \mathrm{d} s \leq 2 C \int_{s}^{t} \int_{\mathbb{R}^{3}}\left(1+|v|^{\gamma+2}\right) f_{s}^{n}(\mathrm{~d} v) \mathrm{d} s$. 
Now for $0 \leq s \leq t \leq T$ with $t-s \leq \varepsilon$, for any $n \geq n_{0}$, any $A>0$, separating the cases $|v| \leq A$ and $|v| \geq A$,

$$
\begin{aligned}
\delta\left(f_{t}^{n}, f_{s}^{n}\right) & \leq 2 C\left(1+A^{\gamma+2}\right)(t-s)+\frac{2 C}{h^{\prime}\left(A^{2}\right)} \int_{s}^{t} \int_{\mathbb{R}^{3}}\left(1+|v|^{\gamma+2}\right) h^{\prime}\left(|v|^{2}\right) f_{s}^{n}(\mathrm{~d} v) \mathrm{d} s \\
& \leq 2 C\left(1+A^{\gamma+2}\right) \varepsilon+\frac{2 C K_{T}}{h^{\prime}\left(A^{2}\right)}
\end{aligned}
$$

because $h^{\prime}$ is nondecreasing and with $K_{T}$ introduced in Step 2. Now for $\eta>0$ fixed, we choose $A_{\eta}>0$ large enough so that $\frac{2 C K_{T}}{h^{\prime}\left(A_{\eta}^{2}\right)} \leq \frac{\eta}{2}$ and conclude that, as soon as $\varepsilon \leq \frac{\eta}{4 C\left(1+A_{\eta}^{\gamma+2}\right)}$, we have $\delta\left(f_{t}^{n}, f_{s}^{n}\right) \leq \eta$ for all $n \geq n_{0}$ and all $s, t \in[0, T]$ such that $|t-s| \leq \varepsilon$.

Step 4. By Step 3, we can find a (not relabelled) subsequence such that $\left(f_{t}^{n}\right)_{t \geq 0}$ converges to a limit $\left(f_{t}\right)_{t \geq 0}$ in $C\left([0, \infty), \mathcal{P}\left(\mathbb{R}^{3}\right)\right)$; this also implies that $\left(f_{t}^{n} \otimes f_{t}^{n}\right)_{t \geq 0}$ tends to $\left(f_{t} \otimes f_{t}\right)_{t \geq 0}$. Hence for all $T>0$, all $\psi \in C_{b}^{2}\left(\mathbb{R}^{3}\right)$ and all $\Psi \in C_{b}^{2}\left(\mathbb{R}^{3} \times \mathbb{R}^{3}\right)$,

$$
\sup _{[0, T]}\left[\left|\int_{\mathbb{R}^{3}} \psi(v)\left(f_{t}^{n}(\mathrm{~d} v)-f_{t}(\mathrm{~d} v)\right)\right|+\left|\int_{\mathbb{R}^{3} \times \mathbb{R}^{3}} \Psi\left(v, v_{*}\right)\left(f_{t}^{n}(\mathrm{~d} v) f_{t}^{n}\left(\mathrm{~d} v_{*}\right)-f_{t}(\mathrm{~d} v) f_{t}\left(\mathrm{~d} v_{*}\right)\right)\right| \rightarrow 0\right.
$$

as $n \rightarrow \infty$. It remains to check that this limit is indeed a weak solution to (1) starting from $f_{0}$.

First, using the uniform integrability property (29),

$$
\sup _{n \geq n_{0}} \sup _{t \in[0, T]} \int_{\mathbb{R}^{3}} h\left(|v|^{2}\right) f_{t}^{n}(\mathrm{~d} v)<\infty
$$

and recalling that $\lim _{r \rightarrow \infty} r^{-1} h(r)=\infty$, one easily check that for all $t \geq 0, m_{2}\left(f_{t}\right)=\lim _{n} m_{2}\left(f_{t}^{n}\right)$. Since now $m_{2}\left(f_{t}^{n}\right)=m_{2}\left(f_{0}^{n}\right) \rightarrow m_{2}\left(f_{0}\right)$, we deduce that $\left(f_{t}\right)_{t \geq 0}$ is energy-conserving as desired.

Next, we fix $\varphi \in C_{b}^{2}\left(\mathbb{R}^{3}\right)$ and recall that $\mathcal{L} \varphi$ is continuous on $\mathbb{R}^{3} \times \mathbb{R}^{3}$ and satisfies the growth bound $\left|\mathcal{L} \varphi\left(v, v_{*}\right)\right| \leq C\left(1+|v|^{2+\gamma}+\left|v_{*}\right|^{2+\gamma}\right)$. We can then let $n \rightarrow \infty$ in the formula

$$
\int_{\mathbb{R}^{3}} \varphi(v) f_{t}^{n}(\mathrm{~d} v)=\int_{\mathbb{R}^{3}} \varphi(v) f_{0}^{n}(\mathrm{~d} v)+\int_{0}^{t} \int_{\mathbb{R}^{3}} \int_{\mathbb{R}^{3}} \mathcal{L} \varphi\left(v, v_{*}\right) f_{s}^{n}\left(\mathrm{~d} v_{*}\right) f_{s}^{n}(\mathrm{~d} v) \mathrm{d} s,
$$

and conclude that (3) is satisfied, using (30) and the uniform integrability given by (29) (recall that $\left.\lim _{r \rightarrow \infty} h^{\prime}(r)=\infty\right)$, i.e.

$$
\sup _{n \geq n_{0}} \int_{0}^{t} \int_{\mathbb{R}^{3}}|v|^{2+\gamma} h^{\prime}\left(|v|^{2}\right) f_{s}^{n}(\mathrm{~d} v) \mathrm{d} s<\infty .
$$

The proof is complete.

\section{Regularity}

We now prove our regularity result Theorem 5 . We begin with the following very mild regularity principle, which guarantees that the hypotheses of Theorem 2 (c) apply at some small time, provided that $f_{0}$ has 4 moments. We then 'bootstrap' to the claimed result, using Theorems 2 and 4 and our uniqueness result.

Lemma 16. Let $\gamma \in(0,1]$ and $f_{0} \in \mathcal{P}_{4}\left(\mathbb{R}^{3}\right)$ be a measure which is not a Dirac mass, and let $\left(f_{t}\right)_{t \geq 0}$ be the weak solution to (11) starting at $f_{0}$. Then, for any $t_{0}>0$, there exists $t_{1} \in\left[0, t_{0}\right)$ such that $f_{t_{1}}$ is not concentrated on a line. 
Proof. If $f_{0}$ is already not concentrated on a line, there is nothing to prove. We thus assume that $f_{0}$ concentrates on a line and, by translational and rotational invariance, that $f_{0}$ concentrates on the $z$-axis $L_{0}=\{(0,0, z): z \in \mathbb{R}\}$. Further, since $f_{0}$ is not a point mass, we can find two disjoint compact intervals $K_{1}, K_{2} \subset L_{0}$ such that $f_{0}\left(K_{1}\right)>0$ and $f_{0}\left(K_{2}\right)>0$.

Step 1. We introduce the following averaged coefficients: for $v \in \mathbb{R}^{3}$ and $f \in \mathcal{P}_{2}\left(\mathbb{R}^{3}\right)$, define

$$
b(v, f)=\int_{\mathbb{R}^{3}} b\left(v-v_{*}\right) f\left(\mathrm{~d} v_{*}\right), \quad a(v, f)=\int_{\mathbb{R}^{3}} a\left(v-v_{*}\right) f\left(\mathrm{~d} v_{*}\right)
$$

and let $\sigma(v, f)$ be a square root of $a(v, f)$. Now, let $\left(B_{t}\right)_{t \geq 0}$ be a 3-dimensional Brownian motion, and $V_{0}$ an independent random variable in $\mathbb{R}^{3}$. From [15, Proposition 10], the Itô stochastic differential equation

$$
V_{t}=V_{0}+\int_{0}^{t} b\left(V_{s}, f_{s}\right) \mathrm{d} s+\int_{0}^{t} \sigma\left(V_{s}, f_{s}\right) \mathrm{d} B_{s}
$$

has a pathwise unique solution and, if $V_{0}$ is $f_{0}$-distributed, then $V_{t} \sim f_{t}$ for all $t \geq 0$. We will denote by $\mathbb{P}_{v_{0}}, \mathbb{E}_{v_{0}}$ the probability and expectation concerning the process started from the deterministic initial condition $V_{0}=v_{0}$. We thus have $f_{t}(A)=\int_{\mathbb{R}^{3}} \mathbb{P}_{v_{0}}\left(V_{t} \in A\right) f_{0}(\mathrm{~d} v)$ for any $A \in \mathcal{B}\left(\mathbb{R}^{3}\right)$, any $t \geq 0$.

Step 2. We now claim that if $F: \mathbb{R}^{3} \rightarrow \mathbb{R}$ is bounded and continuous and $Z \sim \mathcal{N}\left(0, I_{3}\right)$, then

$$
\lim _{\varepsilon \rightarrow 0} \sup _{v_{0} \in K_{1}}\left|\mathbb{E}_{v_{0}}\left[F\left(\frac{V_{\varepsilon}-v_{0}}{\sqrt{\varepsilon}}\right)\right]-\mathbb{E}\left[F\left(\sigma\left(v_{0}, f_{0}\right) Z\right)\right]\right|=0 .
$$

Let $U$ be an open ball containing $K_{1}$, and for $v \in \mathbb{R}^{3}$, let $\pi(v)$ be the unique minimiser of $|v-\tilde{v}|$ over $\tilde{v} \in \bar{U}$. Recalling the growth bounds

$$
\left|b\left(v-v_{*}\right)\right| \leq C\left|v-v_{*}\right|^{1+\gamma}, \quad\left\|a\left(v-v_{*}\right)\right\| \leq C\left|v-v_{*}\right|^{2+\gamma},
$$

that $\sup _{t \geq 0} m_{4}\left(f_{t}\right)<\infty$ by Theorem 2 (a), one checks that $\left|b\left(v, f_{s}\right)\right|+\left\|\sigma\left(f_{s}, v\right)\right\| \leq C\left(1+|v|^{1+\gamma}\right)$ and, since $f_{t} \rightarrow f_{0}$ weakly as $t \rightarrow 0$, that $a\left(v, f_{t}\right) \rightarrow a\left(v, f_{0}\right)$, and thus $\sigma\left(v, f_{t}\right) \rightarrow \sigma\left(v, f_{0}\right)$, uniformly over $v \in \bar{U}$, as $t \rightarrow 0$. We now define

$$
b_{t}(v)=b\left(\pi(v), f_{t}\right) ; \quad \sigma_{t}(v)=\sigma\left(\pi(v), f_{t}\right)
$$

so that $b_{t}(v)$ and $\sigma_{t}(v)$ are bounded, globally Lipschitz in $v$, agree with $b\left(v, f_{t}\right), \sigma\left(v, f_{t}\right)$ for $v \in \bar{U}$ and $\sigma_{t}(v)$ converges uniformly on $\mathbb{R}^{3}$ as $t \downarrow 0$. Now, let $\tilde{V}_{t}$ be the solution to the stochastic differential equation (31) with these coefficients in place of $b\left(v, f_{t}\right)$ and $\sigma\left(v, f_{t}\right)$, and let $T$ be the stopping time when $\tilde{V}_{t}$ first leaves $U$. By uniqueness, we have $V_{t}=\tilde{V}_{t}$ for all $t \in[0, T]$. Using now that $b_{t}$ and $\sigma_{t}$ are bounded, that $\sigma_{t} \rightarrow \sigma_{0}$ uniformly and that $\tilde{V}_{t} \rightarrow v_{0}$ as $t \rightarrow 0$, we see that

$$
\begin{aligned}
& \limsup _{\varepsilon \rightarrow 0} \sup _{v_{0} \in K_{1}} \mathbb{E}_{v_{0}}\left[\left|\frac{\tilde{V}_{\varepsilon}-v_{0}}{\sqrt{\varepsilon}}-\sigma_{0}\left(v_{0}\right) \frac{B_{\varepsilon}}{\sqrt{\varepsilon}}\right|^{2}\right] \\
\leq & \limsup _{\varepsilon \rightarrow 0} \sup _{v_{0} \in K_{1}} \frac{1}{\varepsilon} \mathbb{E}_{v_{0}}\left[2\left(\int_{0}^{\varepsilon} b_{s}\left(\tilde{V}_{s}\right) \mathrm{d} s\right)^{2}+2\left(\int_{0}^{\varepsilon}\left(\sigma_{s}\left(\tilde{V}_{s}\right)-\sigma_{0}\left(v_{0}\right)\right) \mathrm{d} B_{s}\right)^{2}\right]=0 .
\end{aligned}
$$

Recalling that $\sigma_{0}\left(v_{0}\right)=\sigma\left(v_{0}, f_{0}\right)$ when $v_{0} \in K_{1}$ and that $\frac{B_{\varepsilon}}{\sqrt{\varepsilon}} \sim \mathcal{N}\left(0, I_{3}\right)$, we conclude that

$$
\begin{aligned}
& \sup _{v_{0} \in K_{1}}\left|\mathbb{E}_{v_{0}}\left[F\left(\frac{V_{\varepsilon}-v_{0}}{\sqrt{\varepsilon}}\right)\right]-\mathbb{E}_{v_{0}}\left[F\left(\sigma\left(v_{0}, f_{0}\right) Z\right)\right]\right| \\
\leq & \sup _{v_{0} \in K_{1}}\left|\mathbb{E}_{v_{0}}\left[F\left(\frac{\tilde{V}_{\varepsilon}-v_{0}}{\sqrt{\varepsilon}}\right)\right]-\mathbb{E}_{v_{0}}\left[F\left(\sigma_{0}\left(v_{0}\right) \frac{B_{\varepsilon}}{\sqrt{\varepsilon}}\right)\right]\right|+2\|F\|_{\infty} \sup _{v_{0} \in K_{1}} \mathbb{P}(T<\varepsilon) \rightarrow 0
\end{aligned}
$$


where the final convergence follows (33) and the fact that $\sup _{v_{0} \in K_{1}} \mathbb{P}(T<\varepsilon) \rightarrow 0$ because $d\left(K_{1}, U^{c}\right)=\inf \left\{|v-\tilde{v}|: v \in K_{1}, \tilde{v} \notin U\right\}>0$ and because $b_{t}$ and $\sigma_{t}$ are bounded. The proof of the claim is complete.

Step 3. We now construct three test functions $F_{i}$ to which apply Step 2: let $B_{i} \subset \mathbb{R}^{2}, i=$ $1,2,3$ be disjoint open balls in the plane such that no line (in the plane) meets all three, and let $\chi_{i}: \mathbb{R}^{2} \rightarrow[0,1]$ be nonzero, smooth bump functions, supported on each $B_{i}$. Now, we define $\rho: \mathbb{R}^{3} \rightarrow \mathbb{R}^{2}$ the projection $\rho\left(v_{1}, v_{2}, v_{3}\right)=\left(v_{1}, v_{2}\right)$. We then introduce the bounded smooth functions $F_{i}: \mathbb{R}^{3} \rightarrow[0,1]$ defined by $F_{i}(v)=\chi_{i}(\rho(v))$. Observe that $F_{i}(v) \leq \mathbb{1}_{\left\{\rho(v) \in B_{i}\right\}}$.

Since $f_{0}$ concentrates on the $z$-axis $L_{0}$, denoting by $e_{3}=(0,0,1)$, we have, for all $v_{0} \in L_{0}$,

$$
a\left(v_{0}, f_{0}\right)=\int_{\mathbb{R}^{3}}\left|v_{0}-v\right|^{\gamma+2} \Pi_{\left(v-v_{0}\right)^{\perp}} f_{0}(\mathrm{~d} v)=h\left(v_{0}\right) \Pi_{e_{3}^{\perp}}=h\left(v_{0}\right)\left(\begin{array}{ccc}
1 & 0 & 0 \\
0 & 1 & 0 \\
0 & 0 & 0
\end{array}\right),
$$

where $h\left(v_{0}\right)=\int_{\mathbb{R}^{3}}\left|v_{0}-v\right|^{\gamma+2} f_{0}(\mathrm{~d} v)$. One easily checks that $h$ is bounded from above and from below on $K_{1}$, since $\sup _{v_{0} \in K_{1}} h\left(v_{0}\right) \leq C\left(1+m_{2+\gamma}\left(f_{0}\right)\right)$ and $\inf _{v_{0} \in K_{1}} h\left(v_{0}\right) \geq \alpha^{\gamma+2} f_{0}\left(K_{2}\right)$, where $\alpha>0$ is the distance between $K_{1}$ and $K_{2}$. Since $\sigma\left(v_{0}, f_{0}\right)=\left[a\left(v_{0}, f_{0}\right)\right]^{1 / 2}$ and since $\rho(Z) \sim \mathcal{N}\left(0, I_{2}\right)$, we deduce that for some $\delta>0$ and all $i=1,2,3$,

$$
\inf _{v_{0} \in K_{1}} \mathbb{E}_{v_{0}}\left[F_{i}\left(\sigma\left(v_{0}, f_{0}\right) Z\right)\right]=\inf _{v_{0} \in K_{1}} \mathbb{E}\left[\chi_{i}\left(h^{1 / 2}\left(v_{0}\right) \rho(Z)\right)\right] \geq 2 \delta>0 .
$$

Thanks to (32), we can find $\varepsilon_{0}>0$ such that for all $\varepsilon \in\left(0, \varepsilon_{0}\right)$, all $i=1,2,3$,

$$
\inf _{v_{0} \in K_{1}} \mathbb{E}_{v_{0}}\left[F_{i}\left(\frac{V_{\varepsilon}-v_{0}}{\sqrt{\varepsilon}}\right)\right] \geq \delta \quad \text { whence } \inf _{v_{0} \in K_{1}} \mathbb{P}_{v_{0}}\left(\rho\left(\frac{V_{\varepsilon}-v_{0}}{\sqrt{\varepsilon}}\right) \in B_{i}\right) \geq \delta .
$$

Step 4. Now, we fix $t_{0}>0$ as in the statement, and consider $t_{1} \in\left(0, \varepsilon_{0} \wedge t_{0}\right)$. For a given line $L=\left\{x_{0}+\lambda u_{0}: \lambda \in \mathbb{R}\right\} \subset \mathbb{R}^{3}$ and for $v_{0} \in K_{1}$, we denote by $L_{t_{1}, v_{0}}=\rho\left(\left(L-v_{0}\right) / \sqrt{t_{1}}\right)$, which is a line (or a point) in $\mathbb{R}^{2}$. There is $i \in\{1,2,3\}$, possibly depending on $t_{1}$ and on $v_{0}$, such that $L_{t_{1}, v_{0}} \cap B_{i}=\emptyset$, so that

$$
\begin{aligned}
\mathbb{P}_{v_{0}}\left(V_{t_{1}} \in L\right) & =\mathbb{P}_{v_{0}}\left(\frac{V_{t_{1}}-v_{0}}{\sqrt{t_{1}}} \in \frac{L-v_{0}}{\sqrt{t_{1}}}\right) \\
& \leq \mathbb{P}_{v_{0}}\left(\rho\left(\frac{V_{t_{1}}-v_{0}}{\sqrt{t_{1}}}\right) \in L_{t_{1}, v_{0}}\right) \\
& \leq 1-\mathbb{P}_{v_{0}}\left(\rho\left(\frac{V_{t_{1}}-v_{0}}{\sqrt{t_{1}}}\right) \in B_{i}\right) \\
& \leq 1-\delta
\end{aligned}
$$

by Step 3. In other words, for all $v_{0} \in K_{1}, \mathbb{P}_{v_{0}}\left(V_{t_{1}} \in \mathbb{R}^{3} \backslash L\right) \geq \delta$, whence

$$
f_{t_{1}}\left(\mathbb{R}^{3} \backslash L\right)=\int_{\mathbb{R}^{3}} \mathbb{P}_{v_{0}}\left(V_{t_{1}} \notin L\right) f_{0}\left(\mathrm{~d} v_{0}\right) \geq \delta f_{0}\left(K_{1}\right)>0 .
$$

The proof is complete.

We now prove our claimed result.

Proof of Theorem 5. Let $f_{0} \in \mathcal{P}_{2}\left(\mathbb{R}^{3}\right)$ not be a point mass, and let $\left(f_{t}\right)_{t \geq 0}$ be any weak solution to (1) starting at $f_{0}$. Fix $t_{0}>0$. By Theorem 2-(a), picking $t_{1} \in\left(0, t_{0}\right)$ arbitrarily, we have $m_{4}\left(f_{t_{1}}\right)<\infty$ and, due to conservation of energy and momentum, $f_{t_{1}}$ is not a point mass. We can therefore apply Lemma 16 to find $t_{2} \in\left[t_{1}, t_{0}\right)$ such that $f_{t_{2}}$ is not concentrated on a line, and we also have $m_{4}\left(f_{t_{2}}\right)<\infty$, still by Theorem 2 (a), because $t_{2}>0$. 
Now, by Theorem 2-(c), there exists $a$ solution $\left(g_{t}\right)_{t \geq 0}$ to (11) starting at $g_{0}=f_{t_{2}}$ such that, for all $s, k \geq 0$ and $\delta>0$,

$$
\sup _{t \geq \delta}\left\|g_{t}\right\|_{H_{s}^{k}\left(\mathbb{R}^{3}\right)}<\infty
$$

and such that $H\left(g_{t}\right)<\infty$ for all $t>0$; by Theorem $4 g_{t}$ is further analytic for all $t>0$.

By uniqueness, see Theorem 8 and recall that $m_{4}\left(f_{t_{2}}\right)<\infty$, there is a unique weak solution to (11) starting at $g_{0}=f_{t_{2}}$, whence $g_{t}=f_{t_{2}+t}$ for all $t \geq 0$. In particular, $f_{t_{0}}=g_{t_{0}-t_{2}}$ is analytic and has finite entropy and (choosing $\delta=t_{0}-t_{2}$ ), for all $s, k \geq 0, \sup _{t \geq t_{0}}\left\|f_{t}\right\|_{H_{s}^{k}\left(\mathbb{R}^{3}\right)}<\infty$.

\section{Proof of the Central inequality}

We finally handle the

Proof of Lemma 13. We introduce the shortened notation $x=v-v_{*}, \tilde{x}=\tilde{v}-\tilde{v}_{*}$ and recall that

$$
\mathcal{A} c_{p, \varepsilon}\left(v, v_{*}, \tilde{v}, \tilde{v}_{*}\right) \leq k_{p, \varepsilon}^{(1)}+k_{p, \varepsilon}^{(2)}+\tilde{k}_{p, \varepsilon}^{(2)}+k_{p, \varepsilon}^{(3)}+\tilde{k}_{p, \varepsilon}^{(3)},
$$

where $k_{p, \varepsilon}^{(1)}=k_{p, \varepsilon}^{(1)}\left(v, v_{*}, \tilde{v}, \tilde{v}_{*}\right), k_{p, \varepsilon}^{(2)}=k_{p, \varepsilon}^{(2)}\left(v, v_{*}, \tilde{v}, \tilde{v}_{*}\right), \tilde{k}_{p, \varepsilon}^{(2)}=k_{p, \varepsilon}^{(2)}\left(\tilde{v}, \tilde{v}_{*}, v, v_{*}\right)$, etc. In the whole proof, $C$ is allowed to change from line to line and to depend (only) on $p$ and $\gamma$.

Step 1. Here we show, and this is the most tedious estimate, that

$$
\begin{aligned}
k_{p, \varepsilon}^{(1)} \leq & 2 c_{p+\gamma, \varepsilon}(v, \tilde{v}) \\
& +C \sqrt{\varepsilon}\left(1+\left|v_{*}\right|^{p}+\left|\tilde{v}_{*}\right|^{p}\right) c_{p+\gamma, \varepsilon}(v, \tilde{v}) \\
& +C \sqrt{\varepsilon}\left(1+|v|^{p}+|\tilde{v}|^{p}\right) c_{p+\gamma, \varepsilon}\left(v_{*}, \tilde{v}_{*}\right) \\
& +\frac{C}{\sqrt{\varepsilon}}\left(1+\left|v_{*}\right|^{p+\gamma}+\left|\tilde{v}_{*}\right|^{p+\gamma}\right) c_{p, \varepsilon}(v, \tilde{v}) \\
& +\frac{C}{\sqrt{\varepsilon}}\left(1+|v|^{p+\gamma}+|\tilde{v}|^{p+\gamma}\right) c_{p, \varepsilon}\left(v_{*}, \tilde{v}_{*}\right) .
\end{aligned}
$$

We start from

$$
k_{p, \varepsilon}^{(1)}=\left(1+|v|^{p}+|\tilde{v}|^{p}\right) \varphi_{\varepsilon}^{\prime}\left(|v-\tilde{v}|^{2}\right)\left[g_{1}+g_{2}+g_{3}\right]
$$

where

$$
\begin{aligned}
& g_{1}=\left[(v-\tilde{v})-\left(v_{*}-\tilde{v}_{*}\right)\right] \cdot(b(x)-b(\tilde{x}))+\|\sigma(x)-\sigma(\tilde{x})\|^{2}, \\
& g_{2}=(v-\tilde{v}) \cdot(b(x)-b(\tilde{x})), \\
& g_{3}=\left(v_{*}-\tilde{v}_{*}\right) \cdot(b(x)-b(\tilde{x})) .
\end{aligned}
$$

Step 1.1. Recalling that $b(x)=-2|x|^{\gamma} x$ and using (12), we find

$$
\begin{aligned}
g_{1} & \leq 2(x-\tilde{x}) \cdot\left[-|x|^{\gamma} x+|\tilde{x}|^{\gamma} \tilde{x}\right]+2|x|^{\gamma+2}+2|\tilde{x}|^{\gamma+2}-4|x|^{\gamma / 2}|\tilde{x}|^{\gamma / 2}(x \cdot \tilde{x}) \\
& =2\left(|x|^{\gamma}+|\tilde{x}|^{\gamma}\right)(x \cdot \tilde{x})-4|x|^{\gamma / 2}|\tilde{x}|^{\gamma / 2}(x \cdot \tilde{x}) \\
& =2(x \cdot \tilde{x})\left(|x|^{\gamma / 2}-|\tilde{x}|^{\gamma / 2}\right)^{2} .
\end{aligned}
$$

Using now (10) with $\alpha=\gamma / 2$,

$$
g_{1} \leq 2|x||\tilde{x}|(|x| \vee|\tilde{x}|)^{\gamma-2}(|x|-|\tilde{x}|)^{2}=2(|x| \wedge|\tilde{x}|)(|x| \vee|\tilde{x}|)^{\gamma-1}(|x|-|\tilde{x}|)^{2} \leq 2(|x| \wedge|\tilde{x}|)^{\gamma}|x-\tilde{x}|^{2} .
$$

Since $|x-\tilde{x}|=\left|(v-\tilde{v})-\left(v_{*}-\tilde{v}_{*}\right)\right|$, we end with

$$
g_{1} \leq 2(|x| \wedge|\tilde{x}|)^{\gamma}|v-\tilde{v}|^{2}+2(|x| \wedge|\tilde{x}|)^{\gamma}\left(2|v-\tilde{v}|\left|v_{*}-\tilde{v}_{*}\right|+\left|v_{*}-\tilde{v}_{*}\right|^{2}\right) .
$$


Step 1.2. We next study $g_{2}$, assuming without loss of generality that $|x| \geq|\tilde{x}|$. We write, using (10) with $\alpha=\gamma$,

$$
\begin{aligned}
g_{2} & =2(v-\tilde{v}) \cdot\left[-|x|^{\gamma}(x-\tilde{x})+\left(|\tilde{x}|^{\gamma}-|x|^{\gamma}\right) \tilde{x}\right] \\
& \leq-2|x|^{\gamma}(v-\tilde{v}) \cdot(x-\tilde{x})+2|v-\tilde{v}||\tilde{x}|(|x| \vee|\tilde{x}|)^{\gamma-1}|| x|-| \tilde{x}|| \\
& \leq-2|x|^{\gamma}(v-\tilde{v}) \cdot(x-\tilde{x})+2|v-\tilde{v}||\tilde{x}|^{\gamma}|x-\tilde{x}| .
\end{aligned}
$$

Since now $x=v-v_{*}$ and $\tilde{x}=\tilde{v}-\tilde{v}_{*}$, we see that

$$
\begin{aligned}
g_{2} & \leq-2|x|^{\gamma}|v-\tilde{v}|^{2}+2|x|^{\gamma}|v-\tilde{v}|\left|v_{*}-\tilde{v}_{*}\right|+2|\tilde{x}|^{\gamma}\left[|v-\tilde{v}|^{2}+|v-\tilde{v}|\left|v_{*}-\tilde{v}_{*}\right|\right] \\
& \leq 2\left(|x|^{\gamma}+|\tilde{x}|^{\gamma}\right)|v-\tilde{v}|\left|v_{*}-\tilde{v}_{*}\right|
\end{aligned}
$$

since $|x| \geq|\tilde{x}|$ by assumption. By symmetry, the same bound holds when $|x| \leq|\tilde{x}|$.

Step 1.3. Using now (11), we see that

$$
g_{3} \leq 2\left|v_{*}-\tilde{v}_{*}\right|\left[|x|^{\gamma}+|\tilde{x}|^{\gamma}\right]|x-\tilde{x}| \leq 2\left(|x|^{\gamma}+|\tilde{x}|^{\gamma}\right)\left[|v-\tilde{v}|\left|v_{*}-\tilde{v}_{*}\right|+\left|v_{*}-\tilde{v}_{*}\right|^{2}\right] .
$$

Step 1.4. Gathering Steps 1.1, 1.2, 1.3, we have checked that

$$
k_{p, \varepsilon}^{(1)} \leq\left(1+|v|^{p}+|\tilde{v}|^{p}\right) \varphi_{\varepsilon}^{\prime}\left(|v-\tilde{v}|^{2}\right)\left[2(|x| \wedge|\tilde{x}|)^{\gamma}|v-\tilde{v}|^{2}+C\left(|x|^{\gamma}+|\tilde{x}|^{\gamma}\right)\left(|v-\tilde{v}|\left|v_{*}-\tilde{v}_{*}\right|+\left|v_{*}-\tilde{v}_{*}\right|^{2}\right)\right] .
$$

Recalling that $r \varphi_{\varepsilon}^{\prime}(r) \leq \varphi_{\varepsilon}(r)$ by (16) and that $|x|^{\gamma} \leq|v|^{\gamma}+\left|v_{*}\right|^{\gamma}$ and $|\tilde{x}|^{\gamma} \leq|\tilde{v}|^{\gamma}+\left|\tilde{v}_{*}\right|^{\gamma}$, we may write $k_{p, \varepsilon}^{(1)} \leq k_{p, \varepsilon}^{(11)}+k_{p, \varepsilon}^{(12)}$, where

$$
\begin{aligned}
& k_{p, \varepsilon}^{(11)}=2\left(1+|v|^{p}+|\tilde{v}|^{p}\right)\left[\left(|v|^{\gamma}+\left|v_{*}\right|^{\gamma}\right) \wedge\left(|\tilde{v}|^{\gamma}+\left|\tilde{v}_{*}\right|^{\gamma}\right)\right] \varphi_{\varepsilon}\left(|v-\tilde{v}|^{2}\right), \\
& k_{p, \varepsilon}^{(12)}=C\left(1+|v|^{p}+|\tilde{v}|^{p}\right)\left(|v|^{\gamma}+\left|v_{*}\right|^{\gamma}+|\tilde{v}|^{\gamma}+\left|\tilde{v}_{*}\right|^{\gamma}\right) \varphi_{\varepsilon}^{\prime}\left(|v-\tilde{v}|^{2}\right)\left(|v-\tilde{v}|\left|v_{*}-\tilde{v}_{*}\right|+\left|v_{*}-\tilde{v}_{*}\right|^{2}\right) .
\end{aligned}
$$

First,

$$
\begin{aligned}
k_{p, \varepsilon}^{(11)} & \leq 2\left(|v|^{\gamma}+\left|v_{*}\right|^{\gamma}\right) \varphi_{\varepsilon}\left(|v-\tilde{v}|^{2}\right)+2|v|^{p}\left(|v|^{\gamma}+\left|v_{*}\right|^{\gamma}\right) \varphi_{\varepsilon}\left(|v-\tilde{v}|^{2}\right)+2|\tilde{v}|^{p}\left(|\tilde{v}|^{\gamma}+\left|\tilde{v}_{*}\right|^{\gamma}\right) \varphi_{\varepsilon}\left(|v-\tilde{v}|^{2}\right) \\
& =2\left(|v|^{p+\gamma}+|\tilde{v}|^{p+\gamma}\right) \varphi_{\varepsilon}\left(|v-\tilde{v}|^{2}\right)+2\left(|v|^{\gamma}+\left|v_{*}\right|^{\gamma}+|v|^{p}\left|v_{*}\right|^{\gamma}+|\tilde{v}|^{p}\left|\tilde{v}_{*}\right|^{\gamma}\right) \varphi_{\varepsilon}\left(|v-\tilde{v}|^{2}\right) \\
& \leq 2\left(|v|^{p+\gamma}+|\tilde{v}|^{p+\gamma}\right) \varphi_{\varepsilon}\left(|v-\tilde{v}|^{2}\right)+C\left(1+\left|v_{*}\right|^{\gamma}+\left|\tilde{v}_{*}\right|^{\gamma}\right)\left(1+|v|^{p}+|\tilde{v}|^{p}\right) \varphi_{\varepsilon}\left(|v-\tilde{v}|^{2}\right) \\
& =2 c_{p+\gamma, \varepsilon}(v, \tilde{v})+C\left(1+\left|v_{*}\right|^{p+\gamma}+\left|\tilde{v}_{*}\right|^{p+\gamma}\right) c_{p, \varepsilon}(v, \tilde{v}) .
\end{aligned}
$$

We next use that $a b \leq \varepsilon^{1 / 2} a^{2}+\varepsilon^{-1 / 2} b^{2}$ to write

$$
\begin{aligned}
k_{p, \varepsilon}^{(12) \leq} \leq & C \sqrt{\varepsilon}\left(1+|v|^{p}+|\tilde{v}|^{p}\right)\left(|v|^{\gamma}+\left|v_{*}\right|^{\gamma}+|\tilde{v}|^{\gamma}+\left|\tilde{v}_{*}\right|^{\gamma}\right) \varphi_{\varepsilon}^{\prime}\left(|v-\tilde{v}|^{2}\right)|v-\tilde{v}|^{2} \\
& +\frac{C}{\sqrt{\varepsilon}}\left(1+|v|^{p}+|\tilde{v}|^{p}\right)\left(|v|^{\gamma}+\left|v_{*}\right|^{\gamma}+|\tilde{v}|^{\gamma}+\left|\tilde{v}_{*}\right|^{\gamma}\right) \varphi_{\varepsilon}^{\prime}\left(|v-\tilde{v}|^{2}\right)\left|v_{*}-\tilde{v}_{*}\right|^{2} \\
\leq & C \sqrt{\varepsilon}\left(1+|v|^{p}+|\tilde{v}|^{p}\right)\left(|v|^{\gamma}+\left|v_{*}\right|^{\gamma}+|\tilde{v}|^{\gamma}+\left|\tilde{v}_{*}\right|^{\gamma}\right) \varphi_{\varepsilon}\left(|v-\tilde{v}|^{2}\right) \\
& +\frac{C}{\sqrt{\varepsilon}}\left(1+|v|^{p}+|\tilde{v}|^{p}\right)\left(|v|^{\gamma}+\left|v_{*}\right|^{\gamma}+|\tilde{v}|^{\gamma}+\left|\tilde{v}_{*}\right|^{\gamma}\right)\left|v_{*}-\tilde{v}_{*}\right|^{2}
\end{aligned}
$$


because $r \varphi_{\varepsilon}^{\prime}(r) \leq \varphi_{\varepsilon}(r)$ and $\varphi_{\varepsilon}^{\prime}(r) \leq 1$ by (16). We carry on with

$$
\begin{aligned}
k_{p, \varepsilon}^{(12) \leq} & C \sqrt{\varepsilon}\left(1+|v|^{p+\gamma}+|\tilde{v}|^{p+\gamma}\right) \varphi_{\varepsilon}\left(|v-\tilde{v}|^{2}\right)+C \sqrt{\varepsilon}\left(1+|v|^{p}+|\tilde{v}|^{p}\right)\left(\left|v_{*}\right|^{\gamma}+\left|\tilde{v}_{*}\right|^{\gamma}\right) \varphi_{\varepsilon}\left(|v-\tilde{v}|^{2}\right) \\
& +\frac{C}{\sqrt{\varepsilon}}\left(1+|v|^{p}+|\tilde{v}|^{p}\right)\left(|v|^{\gamma}+\left|v_{*}\right|^{\gamma}+|\tilde{v}|^{\gamma}+\left|\tilde{v}_{*}\right|^{\gamma}\right)\left(1+\varepsilon\left|v_{*}-\tilde{v}_{*}\right|^{2}\right) \varphi_{\varepsilon}\left(\left|v_{*}-\tilde{v}_{*}\right|^{2}\right) \\
\leq & C \sqrt{\varepsilon} c_{p+\gamma, \varepsilon}(v, \tilde{v})+C \sqrt{\varepsilon}\left(1+\left|v_{*}\right|^{\gamma}+\left|\tilde{v}_{*}\right|^{\gamma}\right) c_{p, \varepsilon}(v, \tilde{v}) \\
& +\frac{C}{\sqrt{\varepsilon}}\left(1+|v|^{p}+|\tilde{v}|^{p}\right)\left(|v|^{\gamma}+\left|v_{*}\right|^{\gamma}+|\tilde{v}|^{\gamma}+\left|\tilde{v}_{*}\right|^{\gamma}\right) \varphi_{\varepsilon}\left(\left|v_{*}-\tilde{v}_{*}\right|^{2}\right) \\
& +C \sqrt{\varepsilon}\left(1+|v|^{p}+|\tilde{v}|^{p}\right)\left(|v|^{\gamma}+\left|v_{*}\right|^{\gamma}+|\tilde{v}|^{\gamma}+\left|\tilde{v}_{*}\right|^{\gamma}\right)\left(\left|v_{*}\right|^{2}+\left|\tilde{v}_{*}\right|^{2}\right) \varphi_{\varepsilon}\left(\left|v_{*}-\tilde{v}_{*}\right|^{2}\right) \\
\leq & C \sqrt{\varepsilon} c_{p+\gamma, \varepsilon}(v, \tilde{v})+C \sqrt{\varepsilon}\left(1+\left|v_{*}\right|^{p+\gamma}+\left|\tilde{v}_{*}\right|^{p+\gamma}\right) c_{p, \varepsilon}(v, \tilde{v}) \\
& +\frac{C}{\sqrt{\varepsilon}}\left(1+|v|^{p+\gamma}+|\tilde{v}|^{p+\gamma}\right)\left(1+\left|v_{*}\right|^{\gamma}+\left|\tilde{v}_{*}\right|^{\gamma}\right) \varphi_{\varepsilon}\left(\left|v_{*}-\tilde{v}_{*}\right|^{2}\right) \\
& +C \sqrt{\varepsilon}\left(1+|v|^{p+\gamma}+|\tilde{v}|^{p+\gamma}\right)\left(\left|v_{*}\right|^{2}+\left|\tilde{v}_{*}\right|^{2}\right) \varphi_{\varepsilon}\left(\left|v_{*}-\tilde{v}_{*}\right|^{2}\right) \\
& +C \sqrt{\varepsilon}\left(1+|v|^{p}+|\tilde{v}|^{p}\right)\left(1+\left|v_{*}\right|^{2+\gamma}+\left|\tilde{v}_{*}\right|^{2+\gamma}\right) \varphi_{\varepsilon}\left(\left|v_{*}-\tilde{v}_{*}\right|^{2}\right) .
\end{aligned}
$$

Since $p \geq 2$, since $\gamma \in(0,1)$ and since $\varepsilon \in(0,1]$, we end with

$$
\begin{aligned}
k_{p, \varepsilon}^{(12) \leq} \leq & C \sqrt{\varepsilon} c_{p+\gamma, \varepsilon}(v, \tilde{v})+C\left(1+\left|v_{*}\right|^{p+\gamma}+\left|\tilde{v}_{*}\right|^{p+\gamma}\right) c_{p, \varepsilon}(v, \tilde{v}) \\
& +\frac{C}{\sqrt{\varepsilon}}\left(1+|v|^{p+\gamma}+|\tilde{v}|^{p+\gamma}\right) c_{p, \varepsilon}\left(v_{*}, \tilde{v}_{*}\right) \\
& +C \sqrt{\varepsilon}\left(1+|v|^{p}+|\tilde{v}|^{p}\right) c_{p+\gamma, \varepsilon}\left(v_{*}, \tilde{v}_{*}\right) .
\end{aligned}
$$

Summing the bounds on $k_{p, \varepsilon}^{(11)}$ and $k_{p, \varepsilon}^{(12)}$ leads us to (35).

Step 2. We next prove that

$$
k_{p, \varepsilon}^{(2)} \leq-p|v|^{p+\gamma} \varphi_{\varepsilon}\left(|v-\tilde{v}|^{2}\right)+C\left(1+\left|v_{*}\right|^{p+\gamma}\right) c_{p, \varepsilon}(v, \tilde{v}),
$$

and this will imply, still allowing $C$ to change from line to line and to depend on $p$, that

$$
\begin{aligned}
k_{p, \varepsilon}^{(2)}+\tilde{k}_{p, \varepsilon}^{(2)} & \leq-p\left(|v|^{p+\gamma}+|\tilde{v}|^{p+\gamma}\right) \varphi_{\varepsilon}\left(|v-\tilde{v}|^{2}\right)+C\left(1+\left|v_{*}\right|^{p+\gamma}+\left|\tilde{v}_{*}\right|^{p+\gamma}\right) c_{p, \varepsilon}(v, \tilde{v}) \\
& =-p\left(c_{p+\gamma}(v, \tilde{v})-\varphi_{\varepsilon}\left(|v-\tilde{v}|^{2}\right)\right)+C\left(1+\left|v_{*}\right|^{p+\gamma}+\left|\tilde{v}_{*}\right|^{p+\gamma}\right) c_{p, \varepsilon}(v, \tilde{v}) \\
& \leq-p c_{p+\gamma}(v, \tilde{v})+C\left(1+\left|v_{*}\right|^{p+\gamma}+\left|\tilde{v}_{*}\right|^{p+\gamma}\right) c_{p, \varepsilon}(v, \tilde{v}),
\end{aligned}
$$

where the equality uses the definition (15) of $c_{p+\gamma, \varepsilon}$, and in the final line we absorb $\varphi\left(|v-\tilde{v}|^{2}\right) \leq$ $c_{p, \varepsilon}(v, \tilde{v})$ into the second term.

By (20)-(21) and by definition of $k_{p, \varepsilon}^{(2)}$, we see that

$$
\begin{aligned}
k_{p, \varepsilon}^{(2)} & \leq \varphi_{\varepsilon}\left(|v-\tilde{v}|^{2}\right)\left[-p|v|^{p+\gamma}+p|v|^{p}\left|v_{*}\right|^{\gamma}+C p^{2}\left(|v|^{p-2+\gamma}\left|v_{*}\right|^{2}+|v|^{p-2}\left|v_{*}\right|^{2+\gamma}\right)\right] . \\
& \leq \varphi_{\varepsilon}\left(|v-\tilde{v}|^{2}\right)\left[-p|v|^{p+\gamma}+C\left(1+\left|v_{*}\right|^{2+\gamma}\right)\left(1+|v|^{p}\right)\right]
\end{aligned}
$$

from which (36) follows. 
Step 3. We finally prove that

$$
\begin{aligned}
k_{p, \varepsilon}^{(3)}+\tilde{k}_{p, \varepsilon}^{(3)} \leq & C\left(1+\left|v_{*}\right|^{p+\gamma}+\left|\tilde{v}_{*}\right|^{p+\gamma}\right) c_{p, \varepsilon}(v, \tilde{v}) \\
& +C\left(1+|v|^{p+\gamma}+|\tilde{v}|^{p+\gamma}\right) c_{p, \varepsilon}\left(v_{*}, \tilde{v}_{*}\right) \\
& +C \sqrt{\varepsilon}\left(1+\left|v_{*}\right|^{p}+\left|\tilde{v}_{*}\right|^{p}\right) c_{p+\gamma, \varepsilon}(v, \tilde{v}) \\
& +C \sqrt{\varepsilon}\left(1+|v|^{p}+|\tilde{v}|^{p}\right) c_{p+\gamma, \varepsilon}\left(v_{*}, \tilde{v}_{*}\right)
\end{aligned}
$$

By symmetry, it suffices to treat the case of $k_{p, \varepsilon}^{(3)}$. Recalling that $|\sigma(x) v| \leq C|x|^{\gamma / 2}|v|\left|v_{*}\right|$ by (14), and that $\|\sigma(x)-\sigma(\tilde{x})\| \leq C\left(|x|^{\gamma / 2}+|\tilde{x}|^{\gamma / 2}\right)|x-\tilde{x}|$ by (13), we directly find

$$
\begin{aligned}
k_{p, \varepsilon}^{(3)} & \leq C|v|^{p-1}\left|v_{*}\right||x|^{\gamma / 2}\left(|x|^{\gamma / 2}+|\tilde{x}|^{\gamma / 2}\right)|x-\tilde{x}||v-\tilde{v}| \varphi_{\varepsilon}^{\prime}\left(|v-\tilde{v}|^{2}\right) \\
& \leq C|v|^{p-1}\left|v_{*}\right|\left(|v|^{\gamma}+|\tilde{v}|^{\gamma}+\left|v_{*}\right|^{\gamma}+\left|\tilde{v}_{*}\right|^{\gamma}\right)\left(|v-\tilde{v}|^{2}+|v-\tilde{v}|\left|v_{*}-\tilde{v}_{*}\right|\right) \varphi_{\varepsilon}^{\prime}\left(|v-\tilde{v}|^{2}\right) \\
& =k_{p, \varepsilon}^{(31)}+k_{p, \varepsilon}^{(32)},
\end{aligned}
$$

where

$$
\begin{aligned}
& k_{p, \varepsilon}^{(31)}=C|v|^{p-1}\left|v_{*}\right|\left(|v|^{\gamma}+|\tilde{v}|^{\gamma}+\left|v_{*}\right|^{\gamma}+\left|\tilde{v}_{*}\right|^{\gamma}\right)|v-\tilde{v}|^{2} \varphi_{\varepsilon}^{\prime}\left(|v-\tilde{v}|^{2}\right), \\
& k_{p, \varepsilon}^{(32)}=C|v|^{p-1}\left|v_{*}\right|\left(|v|^{\gamma}+|\tilde{v}|^{\gamma}+\left|v_{*}\right|^{\gamma}+\left|\tilde{v}_{*}\right|^{\gamma}\right)|v-\tilde{v}|\left|v_{*}-\tilde{v}_{*}\right| \varphi_{\varepsilon}^{\prime}\left(|v-\tilde{v}|^{2}\right) .
\end{aligned}
$$

Since $r \varphi_{\varepsilon}^{\prime}(r) \leq \varphi_{\varepsilon}(r)$ by (16), we have

$$
\begin{aligned}
k_{p, \varepsilon}^{(31)} & \leq C\left(1+\left|v_{*}\right|^{1+\gamma}+\left|\tilde{v}_{*}\right|^{1+\gamma}\right)\left(1+|v|^{p-1+\gamma}+|\tilde{v}|^{p-1+\gamma}\right) \varphi_{\varepsilon}\left(|v-\tilde{v}|^{2}\right) \\
& \leq C\left(1+\left|v_{*}\right|^{p+\gamma}+\left|\tilde{v}_{*}\right|^{p+\gamma}\right) c_{p, \varepsilon}(v, \tilde{v}) .
\end{aligned}
$$

Next, we use that, with $a=|v-\tilde{v}|$ and $a_{*}=\left|v_{*}-\tilde{v}_{*}\right|$, since $a\left[\varphi_{\varepsilon}^{\prime}\left(a^{2}\right)\right] \leq \sqrt{a^{2} \varphi_{\varepsilon}^{\prime}\left(a^{2}\right)} \leq \sqrt{\varphi_{\varepsilon}\left(a^{2}\right)}$ by (16),

$$
a a_{*} \varphi_{\varepsilon}^{\prime}\left(a^{2}\right) \leq \sqrt{\varphi_{\varepsilon}\left(a^{2}\right)} \sqrt{\varphi_{\varepsilon}\left(a_{*}^{2}\right)\left(1+\varepsilon a_{*}^{2}\right)} \leq\left[\varphi_{\varepsilon}\left(a^{2}\right)+\varphi_{\varepsilon}\left(a_{*}^{2}\right)\right]\left(1+\sqrt{\varepsilon} a_{*}\right)
$$

to write $k_{p, \varepsilon}^{(32)} \leq k_{p, \varepsilon}^{(321)}+k_{p, \varepsilon}^{(322)}+k_{p, \varepsilon}^{(323)}+k_{p, \varepsilon}^{(324)}$, where

$$
\begin{aligned}
& k_{p, \varepsilon}^{(321)}=C|v|^{p-1}\left|v_{*}\right|\left(|v|^{\gamma}+|\tilde{v}|^{\gamma}+\left|v_{*}\right|^{\gamma}+\left|\tilde{v}_{*}\right|^{\gamma}\right) \varphi_{\varepsilon}\left(|v-\tilde{v}|^{2}\right), \\
& k_{p, \varepsilon}^{(322)}=C|v|^{p-1}\left|v_{*}\right|\left(|v|^{\gamma}+|\tilde{v}|^{\gamma}+\left|v_{*}\right|^{\gamma}+\left|\tilde{v}_{*}\right|^{\gamma}\right) \varphi_{\varepsilon}\left(\left|v_{*}-\tilde{v}_{*}\right|^{2}\right), \\
& k_{p, \varepsilon}^{(323)}=C \sqrt{\varepsilon}|v|^{p-1}\left|v_{*}\right|\left(|v|^{\gamma}+|\tilde{v}|^{\gamma}+\left|v_{*}\right|^{\gamma}+\left|\tilde{v}_{*}\right|^{\gamma}\right)\left|v_{*}-\tilde{v}_{*}\right| \varphi_{\varepsilon}\left(|v-\tilde{v}|^{2}\right), \\
& k_{p, \varepsilon}^{(324)}=C \sqrt{\varepsilon}|v|^{p-1}\left|v_{*}\right|\left(|v|^{\gamma}+|\tilde{v}|^{\gamma}+\left|v_{*}\right|^{\gamma}+\left|\tilde{v}_{*}\right|^{\gamma}\right)\left|v_{*}-\tilde{v}_{*}\right| \varphi_{\varepsilon}\left(\left|v_{*}-\tilde{v}_{*}\right|^{2}\right) .
\end{aligned}
$$

We have

$$
\begin{aligned}
k_{p, \varepsilon}^{(321)} & \leq C\left(1+\left|v_{*}\right|^{1+\gamma}+\left|\tilde{v}_{*}\right|^{1+\gamma}\right)\left(1+|v|^{p-1+\gamma}+|\tilde{v}|^{p-1+\gamma}\right) \varphi_{\varepsilon}\left(|v-\tilde{v}|^{2}\right) \\
& \leq C\left(1+\left|v_{*}\right|^{p+\gamma}+\left|\tilde{v}_{*}\right|^{p+\gamma}\right) c_{p, \varepsilon}(v, \tilde{v}),
\end{aligned}
$$

as well as

$$
\begin{aligned}
k_{p, \varepsilon}^{(322)} & \leq C\left(1+\left|v_{*}\right|^{1+\gamma}+\left|\tilde{v}_{*}\right|^{1+\gamma}\right)\left(1+|v|^{p-1+\gamma}+|\tilde{v}|^{p-1+\gamma}\right) \varphi_{\varepsilon}\left(\left|v_{*}-\tilde{v}_{*}\right|^{2}\right) \\
& \leq C\left(1+|v|^{p+\gamma}+|\tilde{v}|^{p+\gamma}\right) c_{p, \varepsilon}\left(v_{*}, \tilde{v}_{*}\right),
\end{aligned}
$$

and, dropping $\sqrt{\varepsilon}$ and using that $\left|v_{*}-\tilde{v}_{*}\right| \leq\left|v_{*}\right|+\left|\tilde{v}_{*}\right|$,

$$
\begin{aligned}
k_{p, \varepsilon}^{(323)} & \leq C\left(1+\left|v_{*}\right|^{2+\gamma}+\left|\tilde{v}_{*}\right|^{2+\gamma}\right)\left(1+|v|^{p-1+\gamma}+|\tilde{v}|^{p-1+\gamma}\right) \varphi_{\varepsilon}\left(|v-\tilde{v}|^{2}\right) \\
& \leq C\left(1+\left|v_{*}\right|^{p+\gamma}+\left|\tilde{v}_{*}\right|^{p+\gamma}\right) c_{p, \varepsilon}(v, \tilde{v}) .
\end{aligned}
$$


Finally, using again the bound $\left|v_{*}-\tilde{v}_{*}\right| \leq\left|v_{*}\right|+\left|\tilde{v}_{*}\right|$,

$$
\begin{aligned}
k_{p, \varepsilon}^{(324)} & \leq C \sqrt{\varepsilon}\left(1+|v|^{p-1+\gamma}+|\tilde{v}|^{p-1+\gamma}\right)\left(1+\left|v_{*}\right|^{2+\gamma}+\left|\tilde{v}_{*}\right|^{2+\gamma}\right) \varphi_{\varepsilon}\left(\left|v_{*}-\tilde{v}_{*}\right|^{2}\right) \\
& \leq C \sqrt{\varepsilon}\left(1+|v|^{p}+|\tilde{v}|^{p}\right) c_{p+\gamma, \varepsilon}\left(v_{*}, \tilde{v}_{*}\right) .
\end{aligned}
$$

Summing the bounds on $k_{p, \varepsilon}^{(31)}, k_{p, \varepsilon}^{(321)}, k_{p, \varepsilon}^{(322)}, k_{p, \varepsilon}^{(323)}$ and $k_{p, \varepsilon}^{(324)}$ ends the step.

Gathering (34), (35), (37) and (38) completes the proof since $\varepsilon \in(0,1]$.

\section{REFERENCES}

[1] Alonso, R., Bagland, V. and Lods, B. Long time dynamics for the Landau-Fermi-Dirac equation with hard potentials. J. Differential Equations, 270 (2021), 596-663.

[2] Alonso, R., Gamba, I.M. and Taskovic, M. Exponentially-tailed regularity and time asymptotic for the homogeneous Boltzmann equation. arXiv:1711.06596.

[3] Arsen'ev, A.A. And Buryak, O.E. On the connection between a solution of the Boltzmann equation and a solution of the Landau-Fokker-Planck equation. Mathematics of the USSR-Sbornik. 69 (1991), 465.

[4] Bobylev, A.V. Moment inequalities for the Boltzmann equation and applications to spatially homogeneous problems. J. Statist. Phys. 88 (1997), 1183-1214.

[5] Carrapatoso, K. Exponential convergence to equilibrium for the homogeneous Landau equation with hard potentials. Bull. Sci. Math. 139 (2015), 777-805.

[6] Carrillo, J.A., Delgadino, M.G., Desvillettes, L. And Wu, J. The Landau equation as a Gradient Flow. arXiv:2007.08591.

[7] Chen, H., Li, W. And Xu, C.J. Gevrey regularity for solution of the spatially homogeneous Landau equation. Acta Math. Sci. Ser. B 29 (2009), 673-686.

[8] Chen, H., Li, W.X. And Xu, C.J. Analytic smoothness effect of solutions for spatially homogeneous Landau equation. J. Differential Equations 248 (2010), 77-94.

[9] Desvillettes, L. Entropy dissipation estimates for the Landau equation in the Coulomb case and applications. J. Funct. Anal. 269 (2015), 1359-1403.

[10] Desvillettes, L. On asymptotics of the Boltzmann equation when the collisions become grazing. Transport Theory Statist. Phys. 21 (1992), 259-276.

[11] Desvillettes and L., Villani, C. On the spatially homogeneous Landau equation for hard potentials, Part I : existence, uniqueness and smothness. Comm. Partial Differential Equations 25 (2000), 179-259.

[12] Desvillettes and L., Villani, C. On the spatially homogeneous Landau equation for hard potentials, Part II: H-Theorem and Applications. Comm. Partial Differential Equations 25 (2000), 261-298.

[13] Fournier, N. Uniqueness of bounded solutions for the homogeneous Landau equation with a Coulomb potential. Comm. Math. Phys. 299 (2010), 765-782.

[14] Fournier, N. And Guérin, H. Well-posedness of the spatially homogeneous Landau equation for soft potentials. J. Funct. Anal. 256 (2009), 2542-2560.

[15] Fournier, N. And Guillin, A. From a Kac-like particle system to the Landau equation for hard potentials and Maxwell molecules. Ann. Sci. Éc. Norm. Supér. 50 (2017), 157-199.

[16] Fournier, N. And Hauray, M. Propagation of chaos for the Landau equation with moderately soft potentials. Ann. Probab. 44 (2016), 3581-3660.

[17] Fournier, N. And Mischler, S. Rate of convergence of the Nanbu particle system for hard potentials and Maxwell molecules. Ann. Probab. 44(1) (2016), 589-627.

[18] Fournier, N. And Mounot, C. On the well-posedness of the spatially homogeneous Boltzmann equation with a moderate angular singularity. Comm. Math. Phys. 289 (2009), 803-824.

[19] Fournier, N. And Perthame, B. Monge-Kantorovich distance for PDEs: the coupling method. EMS Surv. Math. Sci. 7 (2020), 1-31.

[20] Fournier, N. On exponential moments of the homogeneous Boltzmann equation for hard potentials without cutoff. arXiv preprint arXiv:2012.02982

[21] Funaki, T, The diffusion approximation of the spatially homogeneous Boltzmann equation Duke Math. J. 52 (1985), 1-23.

[22] Golse, F., Imbert, C., Mounot, C. And Vasseur, A. Harnack inequality for kinetic Fokker-Planck equations with rough coefficients and application to the Landau equation. Ann. Sc. Norm. Super. Pisa Cl. Sci. (5) 19 (2019), 253-295. 
[23] Goudon, T. On Boltzmann Equations and Fokker-Planck Asymptotics: Influence of Grazing Collisions. $J$. Statist. Phys. 89 (1997), 751-776.

[24] GuÉRIn, H. Existence and regularity of a weak function-solution for some Landau equations with a stochastic approach. Stochastic Process. Appl. 101 (2002), 303-325.

[25] GuÉrin, H. Solving Landau equation for some soft potentials through a probabilistic approach. Ann. Appl. Probab. 13 (2003), 515-539.

[26] Guo, Y. The Landau equation in a periodic box, Comm. Math. Phys. 231 (2002), 391-434.

[27] He, L. And Yang, X. Well-posedness and asymptotics of grazing collisions limit of Boltzmann equation with Coulomb interaction. SIAM J. Math. Anal. 46 (2014), 4104-4165.

[28] Heydecker, D. Pathwise convergence of the hard spheres Kac process. Ann. Appl. Probab. 29 (2019), 30623127.

[29] Heydecker, D. Kac's Process with Hard Potentials and a Moderate Angular Singularity. arXiv:2008.12943

[30] KAC, M. Foundations of kinetic theory. Proceedings of the Third Berkeley Symposium on Mathematical Statistics and Probability, 1954-1955, vol. III, University of California Press, 171-197.

[31] Mischler, S. And Mounot, C. Kac's Program in Kinetic Theory. Invent. Math. 193 (2013), 1-147.

[32] Mischler, S., Mounot, C. And Wennberg, B. A new approach to quantitative propagation of chaos for drift, diffusion and jump processes. Probab. Theory Related Fields 161 (2015), 1-59.

[33] Mischler, S and Wennberg, B. On the spatially homogeneous Boltzmann equation. Ann. Inst. H. Poincaré Anal. Non Linéaire 16 (1999), 467-501.

[34] Morimoto, Y., Pravda-Starov, K. and Xu, C.J. A remark on the ultra-analytic smoothing properties of the spatially homogeneous Landau equation. Kinet. Relat. Models 6 (2013), 715-727.

[35] Mоuнот, C. Explicit coercivity estimates for the linearized Boltzmann and Landau operators. Comm. Partial Differential Equations 31 (2006), 1321-1348.

[36] Norris, J. A consistency estimate for Kac's model of elastic collisions in a dilute gas. Ann. Appl. Probab. 26 (2016), 1029-1081.

[37] Povzner, A. JA. On the Boltzmann equation in the kinetic theory of gases. Mat. Sb. (N.S.) 58 (1962), 65-86.

[38] TANaKA, H., Probabilistic treatment of the Boltzmann equation of Maxwellian molecules. Z. Wahrsch. und Verw. Gebiete 46 (1978/79), 67-105.

[39] Villani, C. On the spatially homogeneous Landau equation for Maxwellian molecules. Math. Models Methods Appl. Sci. 8 (1998), 957-983.

[40] Villani, C., On a new class of weak solutions to the spatially homogeneous Boltzmann and Landau equations. Arch. Rational Mech. Anal. 143 (1998), 273-307.

[41] Villani, C. A review of mathematical topics in collisional kinetic theory. Handbook of mathematical fluid dynamics Vol. I, 71-305, North-Holland, Amsterdam, 2002.

[42] Villani, C. Topics in Optimal Transportation. American Mathematical Society, No. 58, 2003.

[43] Walsh, J.B. An introduction to stochastic partial differential equations. École d'été de Probabilités de SaintFlour XIV, Lect. Notes in Math. 1180, 265-437, 1986.

N. Fournier : Sorbonne Université, LPSM-UMr 8001, CAse courrier 158,75252 Paris Cedex 05, France. Email address: nicolas.fournier@sorbonne-universite.fr

D. Heydecker : University of Cambridge, Centre for Mathematical Sciences, Wilberforce Road, CB30WA, United Kingdom.

Email address: dh489@cam.ac.uk. 